\title{
Roles of the CIC chloride channel CLH-1 in food-associated salt chemotaxis behavior of C. elegans
}

\author{
Chanhyun Park ${ }^{1 \dagger}$, Yuki Sakurai ${ }^{1}$, Hirofumi Sato ${ }^{1}$, Shinji Kanda ${ }^{1,2}$, Yuichi lino ${ }^{1 *}$, \\ Hirofumi Kunitomo ${ }^{1 *}$
}

${ }^{1}$ Department of Biological Sciences, School of Science, The University of Tokyo, Tokyo, Japan; ${ }^{2}$ Laboratory of Physiology, Atmosphere and Ocean Research Institute, The University of Tokyo, Chiba, Japan

\begin{abstract}
The ability of animals to process dynamic sensory information facilitates foraging in an ever-changing environment. However, molecular and neural mechanisms underlying such ability remain elusive. The $\mathrm{CIC}$ anion channels/transporters play a pivotal role in cellular ion homeostasis across all phyla. Here, we find a $\mathrm{ClC}$ chloride channel is involved in salt concentration chemotaxis of Caenorhabditis elegans. Genetic screening identified two altered-function mutations of $c l h-1$ that disrupt experience-dependent salt chemotaxis. Using genetically encoded fluorescent sensors, we demonstrate that $\mathrm{CLH}-1$ contributes to regulation of intracellular anion and calcium dynamics of salt-sensing neuron, ASER. The mutant CLH-1 reduced responsiveness of ASER to salt stimuli in terms of both temporal resolution and intensity, which disrupted navigation strategies for approaching preferred salt concentrations. Furthermore, other $\mathrm{ClC}$ genes appeared to act redundantly in salt chemotaxis. These findings provide insights into the regulatory mechanism of neuronal responsivity by $\mathrm{ClCs}$ that contribute to modulation of navigation behavior.
\end{abstract}

*For correspondence:

kunitomo@bs.s.u-tokyo.ac.jp

Present address: ${ }^{\dagger}$ Center for Nanomedicine, Institute for Basic Science (IBS), Seoul, Republic of Korea

Competing interest: See page 22

Funding: See page 22

Received: 03 February 2020

Accepted: 04 January 2021

Published: 25 January 2021

Reviewing editor: Piali Sengupta, Brandeis University, United States

(c) Copyright Park et al. This article is distributed under the terms of the Creative Commons Attribution License, which permits unrestricted use and redistribution provided that the original author and source are credited.

\section{Introduction}

Generating an optimal foraging behavior based on experiences is basic and important ability for survival. Mechanisms of food-associated learning have long been addressed in many species, dating back to Pavlovian appetitive conditioning demonstrated in dogs (Braubach et al., 2009; Cho et al., 2016; Gottfried et al., 2003; Hirano et al., 2013; O'Doherty et al., 2003; Otis et al., 2017; Pavlov, 1927; Sasakura and Mori, 2013; Winter and Stich, 2005). By virtue of its simple nervous system and amenability to genetic manipulations, the soil nematode Caenorhabditis elegans has been used to unveil molecular and neural mechanisms of behavior. C. elegans shows food-associated behavioral plasticity in combination with various sensory modalities including gustatory, olfactory, thermosensory, and mechanosensory cues (Colbert and Bargmann, 1995; Hedgecock and Russell, 1975; Kindt et al., 2007; Saeki et al., 2001). We have previously reported that $C$. elegans shows plasticity in chemotaxis to salt (sodium chloride; $\mathrm{NaCl}$ ); wild-type animals are attracted to the salt concentration at which they have been fed, while avoid the concentrations at which they have been starved (salt concentration chemotaxis, Kunitomo et al., 2013, Figure 1a). C. elegans senses inorganic ions mainly through the bilateral salt-sensing neuron pair, ASE (Bargmann and Horvitz, 1991). Sensory input to ASE-right (ASER) is essential and sufficient for food-associated salt chemotaxis. The interneurons postsynaptic to ASER, namely, AIA, AIB, and AIY, regulates exploratory behaviors (Gray et al., 2005; Li et al., 2014; Piggott et al., 2011). Modulation of synaptic transmission between ASER and these interneurons is involved in modification of salt chemotaxis (Kunitomo et al., 2013; Luo et al., 2014; Wang et al., 2017). 
a

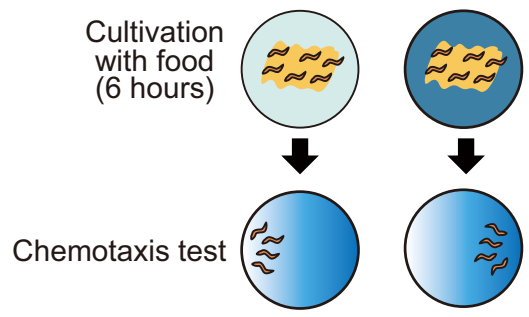

b

$$
\square 0 \mathrm{mM} / \text { Fed } \square 50 \mathrm{mM} / \text { Fed } \square 100 \mathrm{mM} / \text { Fed }
$$
$\square 0 \mathrm{mM} / \mathrm{Stv} \square 50 \mathrm{mM} / \mathrm{Stv} \square 100 \mathrm{mM} / \mathrm{Stv}$

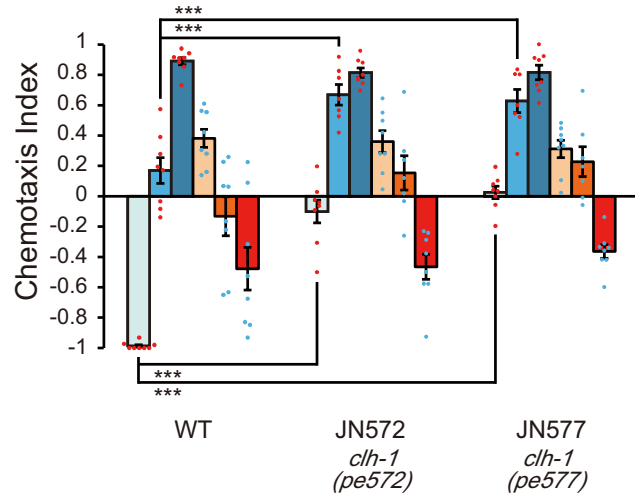

d

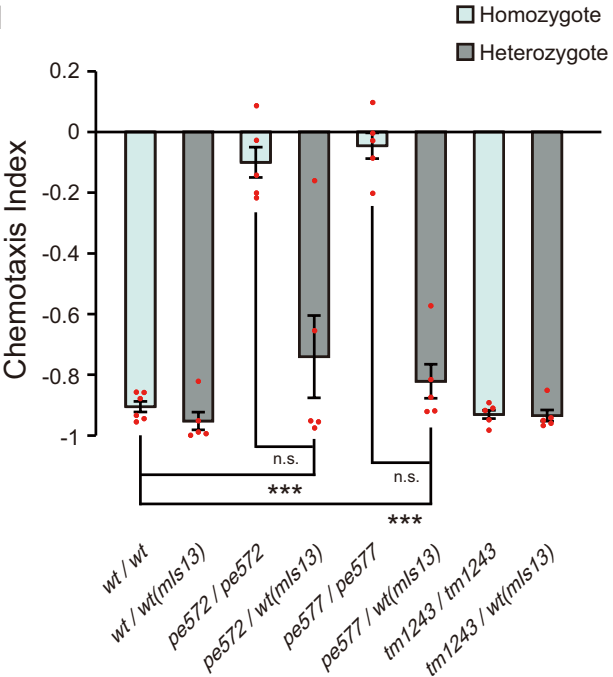

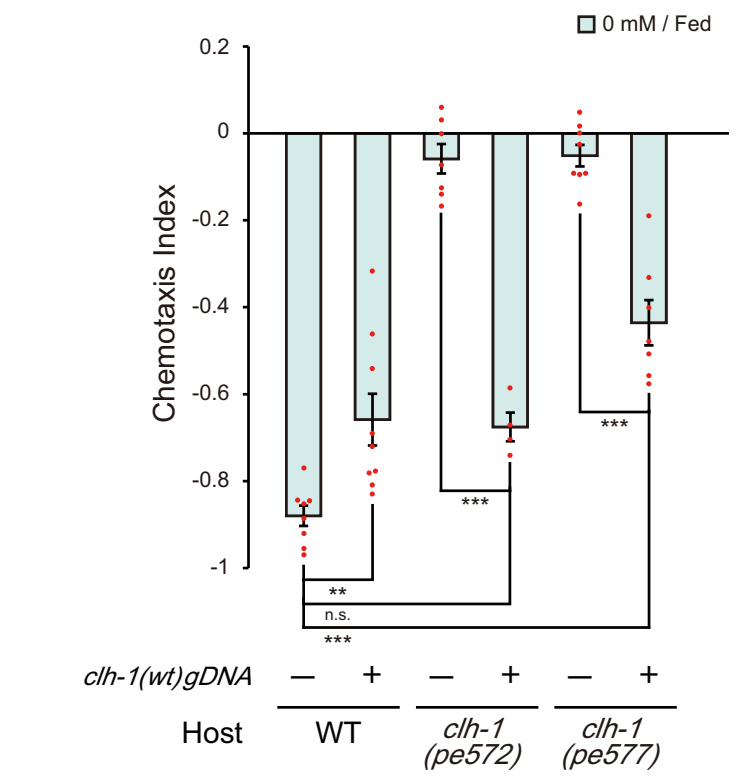

(tivation

without food

(6 hours)

Chemotaxis test
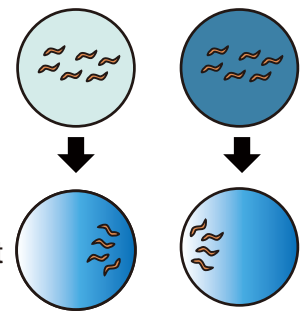

High

Low

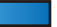

( 2$)$ 
Figure 1 continued

the error bars represent mean +/- s.e.m., $n=8$ assays, Dunnett's test, ${ }^{\star \star \star} p<0.001$. (c) Rescue of clh-1(pe572) and clh-1(pe577) mutants by a clh-1 genomic DNA fragment. Dots represent individual trials. Bars and the error bars represent mean +/- s.e.m., $n \geqq 4$, Tukey's test, ${ }^{* * *} p<0.001,{ }^{* *} p<0.01$, n.s. not significant. (d) Chemotaxis of clh-1 heterozygotes. clh-1 homozygotes were crossed with a clh-1(wt) reporter strain that express GFP in pharyngeal muscle (m/s13). Resulted F1 animals were used for assay. Dots represent individual trials. Bars and the error bars represent mean $+/-$ s.e.m., $n \geqq 4$, Tukey's test, ${ }^{\star \star *} p<0.001$, n.s. not significant.

The online version of this article includes the following figure supplement(s) for figure 1:

Figure supplement 1. Isolation and characterization of salt chemotaxis mutants JN572 and JN577.

Figure supplement 2. Missense mutations in clh-1 responsible for salt chemotaxis defect.

Figure supplement 3. Characterization of clh-1 mutants.

Molecular mechanisms for perception and propagation of salt stimuli in ASER have been proposed. A receptor-type guanylyl cyclase GCY-22 (also known as CHE-5) plays a pivotal role in perception of salt stimuli and is suggested to act as an ion receptor of ASER (Adachi et al., 2010; Kunitomo and lino, 2020; Ortiz et al., 2009; Smith et al., 2013). Excitation of ASER depends also on cyclic nucleotide-gated (CNG) channels consisting of TAX-2 and TAX-4 (Suzuki et al., 2008). C. elegans genome does not contain typical voltage-gated sodium channel genes (Goodman et al., 1998). Instead, voltage-gated calcium channels are responsible for propagation of depolarization, which is examined by electrophysiological studies (Goodman et al., 1998; Shindou et al., 2019). However, contribution of anions to the regulation of ASER activity has not been discussed yet.

Anion transporters play critical roles in regulating excitability of neurons as they finely tune electrophysiological properties of membranes. However, how and which molecules contribute to responsivity of specific neurons and eventually produces behavioral output, remains rudimentary. Here, we identify the $\mathrm{CIC}$ chloride channel CLH-1 as a possible regulator of food-associated salt chemotaxis in C. elegans. CIC proteins transport univalent anions across membranes to control electrochemical potential of excitable cells and to maintain ionic milieu as well as $\mathrm{pH}$ of intracellular organelles (Ahnert-Hilger and Jahn, 2011; Bösl et al., 2001; Branicky et al., 2014). Malfunction of $\mathrm{CIC}$ genes result in various diseases such as myotonia, leukodystrophy, hyperaldosteronism, and epilepsy in humans (Blanz et al., 2007; Charlet-B et alı, 2002; Fernandes-Rosa et al., 2018; Yamamoto et al., 2015). The causal relationship among $\mathrm{ClC}$ malfunction, physiological consequences, and disease manifestations are not fully understood in many cases. CLH-1 shares the highest (37\%) identity with mammalian CIC-2. Functional studies using heterologous expression in Xenopus oocytes and mammalian cells demonstrated that both $\mathrm{CLH}-1$ and $\mathrm{ClC}-2$ are inwardly rectifying chloride channels (Grant et al., 2015; Nehrke et al., 2000; Staley et alı, 1996; Thiemann et al., 1992). On the other hand, two recent studies showed that $\mathrm{ClC}-2$ contributes to $\mathrm{Cl}^{-}$influx in neurons depending on the electrochemical potential of $\mathrm{Cl}^{-}$(Ratté and Prescott, 2011; Rinke et al., 2010). Thus, the function of $\mathrm{CLH}-1 / \mathrm{ClC}-2$ in the nervous system remains elusive.

In this study, we show two novel missense mutations in clh-1 change $\mathrm{NaCl}$ concentration preference of $C$. elegans only after food experience. Genetic analyses revealed that CLH-1 acts in ASER and that both quantitative and anatomical localization of $\mathrm{CLH}-1$ is required for normal chemotaxis. Functional imaging of neurons indicated that mutations in clh-1 altered responsivity of the ASER and AIB neurons, which consequently affect behavioral outputs. These results suggest that responsivity of the salt circuit is maintained by $\mathrm{CLH}-1$ to generate proper navigation behavior in salt chemotaxis.

\section{Results}

\section{Missense mutations of clh-1 give rise to a disorder in food-associated salt concentration chemotaxis}

C. elegans adults cultivated at a particular $\mathrm{NaCl}$ concentration for $6 \mathrm{hr}$ are attracted to the salt concentration if they have been fed, while avoid the concentration if they have been starved (Figure 1a and Figure 1-figure supplement 1a). To better understand molecular mechanisms of salt chemotaxis plasticity, we screened for mutants that showed defects in salt chemotaxis after feeding but not after starvation (see Materials and methods, Figure 1-figure supplement 1b). Two mutants, JN572 and JN577, showed a similar phenotype: an unbiased salt preference after feeding on $\mathrm{NaCl}$ - 
free nematode growth medium (hereinafter referred to as cultivation at $0 \mathrm{mM} \mathrm{NaCl}$ ). Chemotaxis to high salt after feeding on $\mathrm{NGM}$ with $100 \mathrm{mM} \mathrm{NaCl}$ (hereinafter referred to as cultivation at $100 \mathrm{mM}$ $\mathrm{NaCl}$ ), and chemotaxis after starvation were comparable to those of wild-type animals (Figure $1 \mathrm{~b}$ and Figure 1-figure supplement 1c). Neither shortened nor extended cultivation at $0 \mathrm{mM} \mathrm{NaCl}$ did not ameliorate the defects (Figure 1-figure supplement 1d,e), suggesting that the impaired chemotaxis is not due to the delay of behavioral modification. Rather, the mutants were unable to generate migration bias toward low salt. Consistent with this idea, the mutants showed a preference for high salt after cultivation at $50 \mathrm{mM} \mathrm{NaCl}$, under which condition wild-type animals showed an unbiased salt preference (Figure $1 \mathrm{~b}$, and see Discussion).

We mapped mutations of JN572 and JN577 between genetic positions 2.82 and 6.12 (cM) on chromosome II (see Materials and methods, Fay and Bender, 2006; Wicks et al., 2001). Genome sequencing revealed that they carried a distinct missense mutation in the clh-1 gene, one of six $\mathrm{ClC}$ channels/transporters in C. elegans. Mutations predicted M293I and I146T substitutions in CLH-1A in JN572 and JN577, respectively, and they are hereinafter referred to as clh-1(pe572) and clh-1 (pe577) (Figure 1-figure supplement 2a,b). Salt chemotaxis defects of the mutants were recovered by a clh-1(wt) genomic fragment, confirming that clh-1 is the responsible gene, although the effect was partial in clh-1(pe577) (Figure 1c). We also noticed that extra copies of clh-1(wt) genomic fragment weakened low-salt chemotaxis of wild type (Figure 1c), implying that overexpression of $\mathrm{CLH}-1$ could disrupt chemotaxis to low salt.

Interestingly, deletion mutants of $c l h-1$, all of which harbor a lesion in the pore-forming transmembrane domain of CLH-1 and hence are putative loss-of-function alleles (Figure 1-figure supplement 2a), showed almost no discernible defect in salt chemotaxis (Figure 1-figure supplement 3a and Figure 1-figure supplement $1 \mathrm{~d}, \mathrm{e})$. These results suggest that chemotaxis defects of clh-1 (pe572) and clh-1(pe577) were caused by an anomalous activity of clh-1. To characterize the nature of clh-1 missense alleles, we observed food-associated salt chemotaxis of heterozygotes. clh-1 (pe572)/clh-1(wt) and clh-1(pe577)/clh-1(wt) showed normal low-salt chemotaxis, demonstrating that both missense alleles are recessive to wild-type allele (Figure 1d). Also, we noted that the effect of both missense alleles are dosage-dependent, that is, clh-1(pe572)/clh-1(tm1243) and clh-1(pe577)/ clh-1(tm1243) showed a modest defect in salt chemotaxis to low salt after cultivation at $0 \mathrm{mM} \mathrm{NaCl}$ (Figure 1-figure supplement 3b). Furthermore, we unexpectedly found that $\mathrm{clh}-1$ (tm1243) conferred a weak resistance to an acetylcholine receptor agonist, levamisole. On the contrary, clh-1 (pe572) or clh-1(pe577) caused an enhanced sensitivity to levamisole if compared with wild type, suggesting these alleles may not be simple reduction-of-function alleles (Figure 1-figure supplement 3c). Together, these data show that $c l h-1$ (pe572) and clh-1(pe577) (hereinafter collectively referred to as $c / h-1(p e))$ are recessive mutations whose salt chemotaxis phenotype appears in a dosage-dependent manner.

Given that salt chemotaxis defects depend on the dosage of clh-1(pe) alleles, we wondered if overexpression of $c / h-1(p e)$ can give rise to salt chemotaxis defect. To examine this possibility, we introduced clh-1(pe) genomic DNA fragments into wild type or clh-1(tm1243) mutants. Overexpression of mutant clh-1 conferred defects in low-salt chemotaxis in both genomic backgrounds, demonstrating that excess $c / h-1(p e)$ override the canonical $c l h-1(w t)$ function (Figure 1-figure supplement 3d). Therefore, excess clh-1, either wild-type or pe alleles, can impair low-salt chemotaxis. We concluded that $c / h-1$ (pe572) and $c l h-1$ (pe577) are atypical neomorphic alleles that disrupt chemotaxis to low salt after cultivation at $0 \mathrm{mM} \mathrm{NaCl}$.

\section{Roles of $\mathrm{C}$. elegans $\mathrm{CIC}$ channel/transporter genes in salt chemotaxis}

All $\mathrm{ClC}$ anion channels/transporters characterized so far function as either homodimers or heterodimers (Accardi, 2015; Stölting et alo, 2014). The C. elegans genome carries $6 \mathrm{ClC}$ genes (clh-1 through clh-4 for anion channel, and clh-5 and clh-6 for anion transporter, predicted from a key amino acid residue and subcellular localization, Figure 2-figure supplement 1a, Schriever et alo, 1999; Nehrke et al., 2000). This raised a possibility that mutant CLH-1 molecules impaired the function of other $\mathrm{CLH}$ gene products by forming heterodimers, thereby resulting in defective salt chemotaxis. To examine this, we observed salt chemotaxis of the mutants whose clh genes were deleted individually or in combinations with clh-1(pe572) or clh-1(tm1243) mutation. Each single mutant except for clh-5(tm6008) showed normal salt chemotaxis under fed conditions (Figure 2-figure supplement $1 \mathrm{~b}$ ). We then generated and tested a series of double (or triple in the case of clh-3 


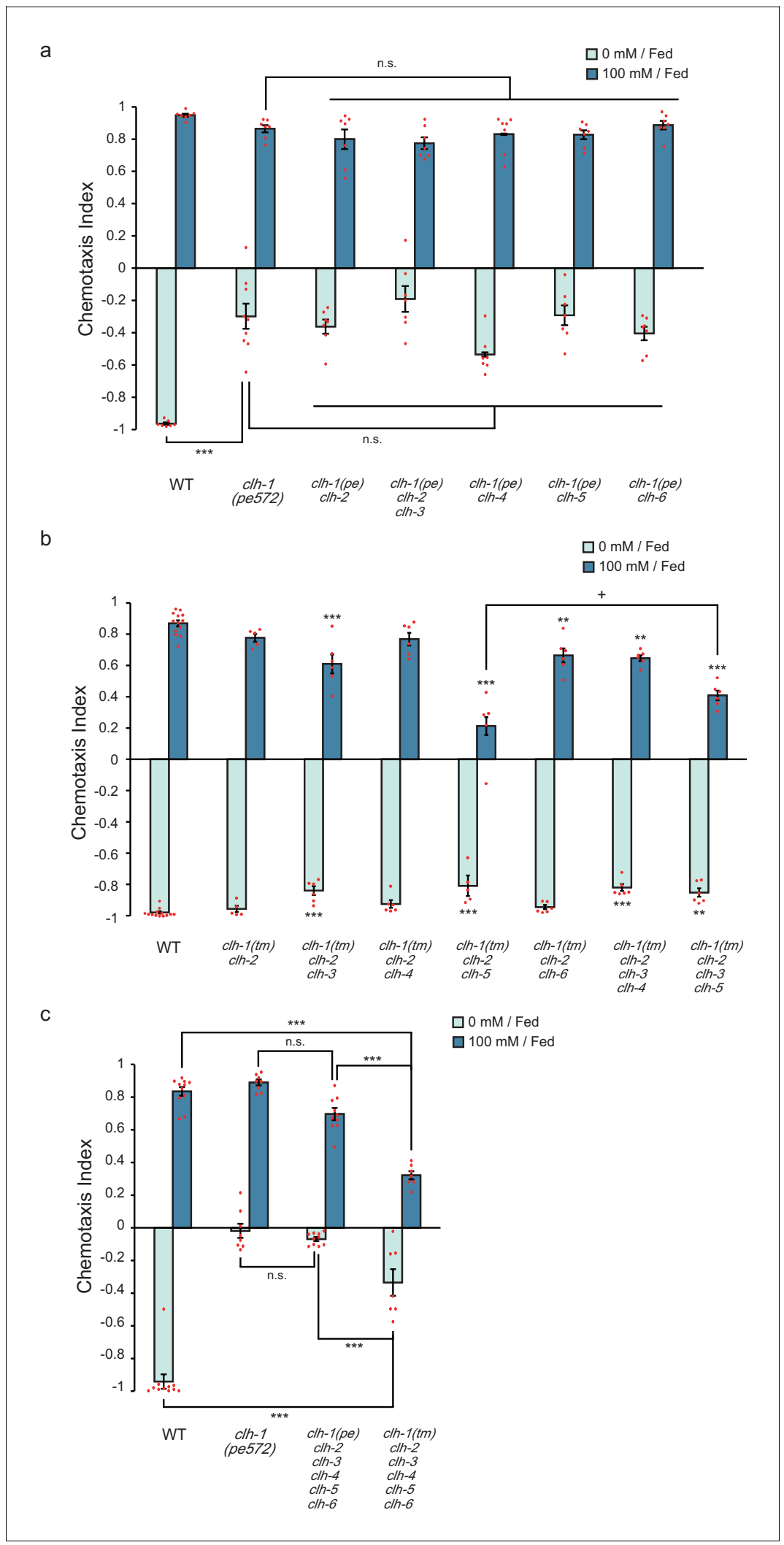

Figure 2. $\mathrm{ClC}$ genes redundantly function in salt chemotaxis. (a) Chemotaxis of clh multiple mutants that carry clh1 (pe572) mutation with a deletion in other clh genes. Dots represent individual trials. Bars and the error bars represent mean + /- s.e.m., $n \geqq 6$ assays, Tukey's test, ${ }^{\star \star \star} p<0.001$, n.s. not significant. (b) Chemotaxis of clh multiple mutants that carry clh-1(tm1243) mutation and a deletion in other clh genes. Dots represent individual Figure 2 continued on next page 
Figure 2 continued

trials. Bars and the error bars represent mean + /- s.e.m., $n \geqq 5$, Tukey's test, ${ }^{\star \star *} p<0.001,{ }^{\star \star} p<0.01$, compared with wild type. $+p<0.05$, compared with indicated mutants. (c) Chemotaxis of $c / h$ hexatruple mutants. Dots represent individual trials. Bars and the error bars represent mean + /- s.e.m., $n \geqq 7$, Tukey's test, ${ }^{\star \star \star} p<0.001$, n.s. not significant.

The online version of this article includes the following figure supplement(s) for figure 2:

Figure supplement 1. Effect of loss of $\mathrm{ClC}$ genes on salt chemotaxis.

(ok763) clh-2(ok636) clh-1(pe572)) mutants that carry clh-1(pe572) and a deletion in another clh gene (Figure 2a). The double mutants showed a defect similar to that of the clh-1(pe572) single mutant, that is, defective chemotaxis toward low salt. A hexatruple mutant that carry clh-1(pe572) and a deletion in five other clh genes also showed similar phenotype (Figure 2c). These results indicate that salt chemotaxis defect of clh-1(pe572) mutant is not attributed to impairment of other CLH proteins.

We next examined whether the clh genes could redundantly act in salt chemotaxis. We started from clh-2(ok636) clh-1(tm1243) double mutants because they had the highest homology (52\% identity) among clh genes. Although the double mutant showed no chemotaxis defect, triple mutants with either clh-3(ok763) or clh-6(tm617) showed an impaired chemotaxis toward high salt (Figure 2b). Triple mutants clh-3(ok763) clh-2(ok636) clh-1(tm1243) and clh-5(tm6008) clh-2(ok636) clh-1(tm1243) showed a weak defect in chemotaxis to low salt. Interestingly, impaired chemotaxis to high salt observed in clh-5 clh-2 clh-1 was partially restored in clh-3(ok763) clh-5(tm6008) clh-2 (ok636) clh-1(tm1243) quadruple mutants (Figure 2b). Many of these multiple mutants and clh-5 (tm6008) single mutants also showed a high immobility index, implying that chemotaxis might be affected by locomotion defects (Figure 2-figure supplement 1c, Figure 2-figure supplement 1d, and see Discussion). Finally, a hexatruple mutant clh-3(ok763) clh-5(tm6008) clh-2(ok636) clh-1 (tm1243); clh-6(tm617); clh-4(ok1162) showed severe defect in both high- and low-salt chemotaxis (Figure 2c). Altogether, our results suggest that clh genes redundantly act in salt chemotaxis. It should be noted that chemotaxis to low salt was more severely affected in clh-1(pe572) hexatruple mutants. This further suggests that the effect of $c / h-1$ (pe572) missense mutation is not simply caused by inhibition of other CLH proteins.

\section{CLH-1 acts in ASER to affect salt preference}

It has previously been reported that clh-1 is expressed in hypodermal cells, seam cells, D-cells of the vulva, and neuronal and glial cells of the head (Grant et al., 2015; Nehrke et al., 2000). These expression patterns were confirmed with a transcriptional reporter (clh-1p::nls4::mTFP, see Materials and methods). Of the head neurons, at least ASE, AWA, and AWC sensory neurons expressed the reporter (Figure 3a). To determine the site of action of clh-1, we performed cell-specific rescue experiments using $c / h-1(w t)$ cDNA. The mutant phenotype was rescued when cDNA was expressed either pan-neuronally or specifically in ASER, suggesting that clh-1 acts in the nervous system including ASER (Figure 3b, and Figure 3-figure supplement 1a for ASER-specific rescue of pe577). On the other hand, clh-1(wt) cDNA failed to rescue clh-1(pe572) when expressed in amphid sheath (AmSh) cells, where CLH-1 function as a pH mediator (Grant et alo, 2015), or in the left-sided ASE neuron (ASEL) (Figure 3b). Unexpectedly, combined expression of the transgene in ASER and AmSh cells or expression in all ciliated neurons also failed to rescue the phenotype (Figure $3 \mathrm{~b}$ ). These phenotypes reminded us of the impairment of low-salt chemotaxis by overexpression of clh-1(wt) genomic fragment (Figure 1c), indicating a possibility that an excess of $\mathrm{CLH}-1(w t)$ in the cells adjacent to ASER could disturb low-salt chemotaxis (see Discussion).

\section{Morphology of ASER and localization of CLH-1 are largely unaffected by missense mutations}

Head sensory neurons of $C$. elegans sense environmental stimuli via neural receptive endings, which are comprised of cilia and microvilli (Perkins et alı, 1986; Ward et alı, 1975; Ware et al., 1975). Function of these endings largely depends on glia which ensheathe them. A recent study showed that AmSh glia regulate the function and shape of the AFD thermosensory neuron's microvilli by 


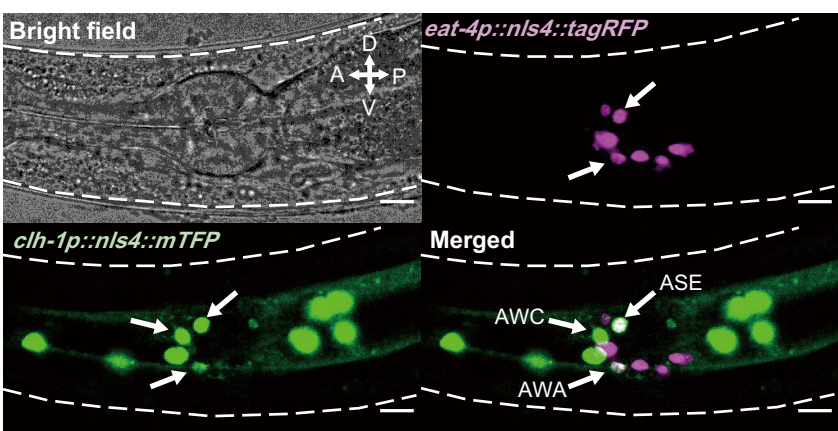

b

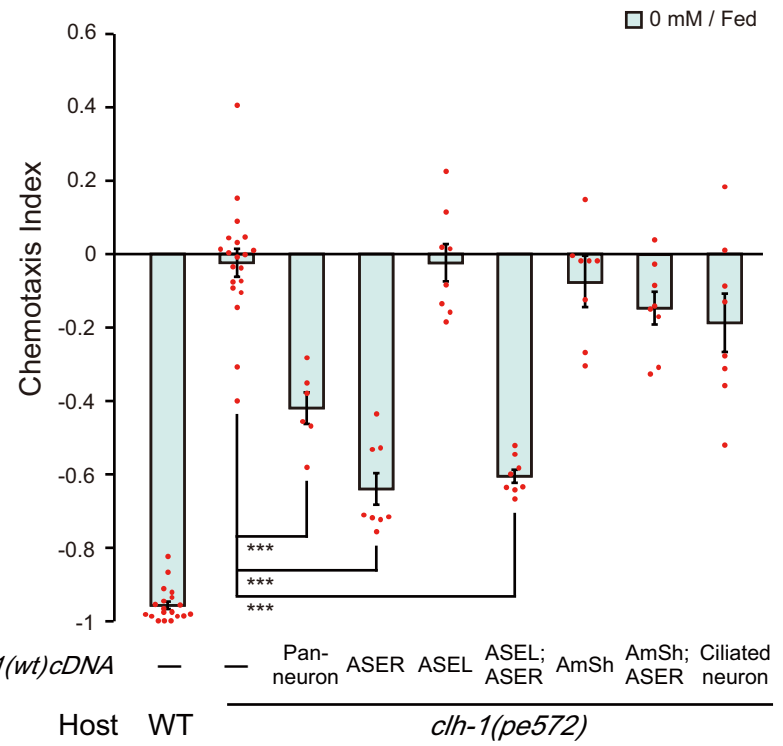

d

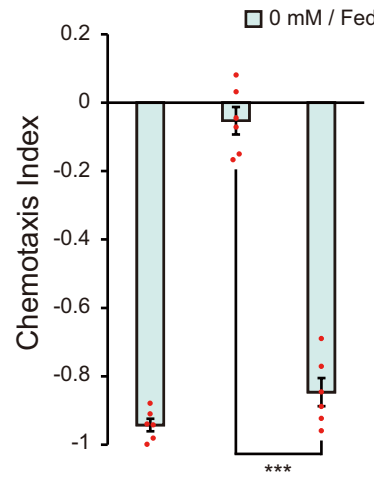

$\mathrm{CLH}-1(w t):: \mathrm{mTFP}$

CLH-1 (pe572)::mTFP

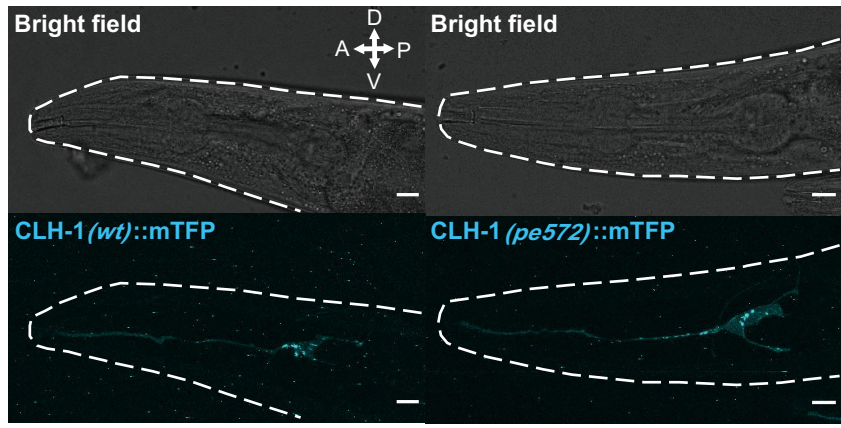

Host WT clh-1(pe572)

Figure 3. clh-1 acts in the salt-sensing neuron ASER. (a) Expression pattern of clh-1p::nls4::mTFP (green, bottom left) in an adult animal. At least three pairs of sensory neurons, AWA, AWC and ASE expressed the marker. eat-4p::n/s4::tagRFP and Dil, that marks glutamatergic neurons (magenta, top right) and six pairs of head sensory neurons (not shown), respectively, were used as position markers for cell identification. Scale bar $=10 \mu \mathrm{m}$. (b) Rescue of clh-1(pe572) mutants by cell-specific expression of clh-1(wt) cDNA. Promoters used in this experiment are as follows; rimb-1p for all neurons, Figure 3 continued on next page 
Figure 3 continued

gcy-5p for ASER, gcy-7p for ASEL, vap-1p for amphid sheath cells, dyf-11p for ciliated neurons. Dots represent individual trials. Bars and the error bars represent mean + /- s.e.m., $n \geqq 6$ assays, Tukey's test. ${ }^{* \star *} p<0.001$. (c) Chemotaxis of clh-1(pe572) mutants that express clh-1(wt)cDNA::mTFP in ASER. mTFP-tagged CLH-1 is functional. Dots represent individual trials. Bars and the error bars represent mean $+/-$ s.e.m., $n=6$, Tukey's test. ${ }^{* \star} p<0.001$. (d) Subcellular localization of CLH-1 in ASER. Panels show gcy-5p:::clh-1(wt)cDNA::mTFP in wild type (left) and gcy-5p::clh-1 (pe572)cDNA::mTFP in clh-1 (pe572) (right). Both CLH-1(wt)::mTFP and CLH-1(pe572)::mTFP localized to the membrane of dendrite, soma, axon, and cell organelles. Scale bar = 10 $\mu \mathrm{m}$.

The online version of this article includes the following figure supplement(s) for figure 3 :

Figure supplement 1. clh-1 acts in ASER in salt chemotaxis, and mutations in clh-1 do not affect morphology of ASER.

modulating efflux of $\mathrm{Cl}^{-}$to the extracellular space of the receptive endings (Singhvi et al., 2016). In addition, it has been elucidated that $\mathrm{CLH}-1$ transports anions to maintain intracellular $\mathrm{pH}$ of $\mathrm{AmSh}$ cells (Grant et al., 2015). These results implied a possibility that the receptive ending of ASER may be impaired in clh-1(pe) mutants. However, we did not find any notable change in the morphology of ASER including its sensory cilium (Figure 3-figure supplement 1b-d). Exposure to different salt and food conditions did not affect the length of ASER sensory cilium both in wild type and in clh-1 mutants, suggesting that the morphology of ASER receptive ending remained largely unchanged (Figure 3-figure supplement 1e).

To examine subcellular localization of $\mathrm{CLH}-1$ in ASER, we generated an mTFP-tagged CLH-1 (CLH-1::mTFP). The fusion protein was functional (Figure 3c). Fluorescent signals were detected in the plasma membrane and cell organelles (Figure 3d, left), as previously reported in mammalian $\mathrm{ClC}$ channels and CIC transporters, respectively (Jentsch, 2008; Jentsch, 2007). Localization patterns of CLH-1::mTFP with M293I mutation were comparable to those of wild type (Figure $3 d$, right). These results indicate that the mutation does not affect intracellular localization of $\mathrm{CLH}-1$. It is unknown whether the $\mathrm{CLH}-1$ (pe) variants are expressed at higher or lower levels than $\mathrm{CLH}-1$ (wt), although expression level of a clh-1 promoter-driven reporter slightly differed between genotypes (Figure 5figure supplement 1e).

\section{Salt stimulus evokes flux of anions in ASER and this response is altered in clh-1(pe) mutants}

Electrophysiological studies using Xenopus oocytes have shown that $\mathrm{CLH}-1$ is an inwardly rectifying channel that conduct $\mathrm{Cl}^{-}$and $\mathrm{HCO}^{-}$, and it is activated by extracellular acidification (Grant et al., 2015; Nehrke et al., 2000). To examine whether the clh-1(pe) mutations affected the property of CLH-1 channel activity, we expressed mutant CLH-1 in Xenopus laevis oocytes and measured wholecell membrane currents via two-electrode voltage clamping. Wild-type $\mathrm{CLH}-1$ showed inwardly rectifying currents from -160 to $-80 \mathrm{mV}$ under the condition with $\mathrm{pH} 7.2$ and $100 \mathrm{mM} \mathrm{NaCl}$ as previously reported (Figure $4 \mathrm{~b}$ and $e$, referred to as $\mathrm{pH} 7 \mathrm{Cl}^{-}$condition, Materials and methods for details, Grant et al., 2015; Nehrke et al., 2000). Small, but similar trend of currents was observed in both CLH-1(M293I) and CLH-1(I146T) (Figure 4c-e). To further characterize the CLH-1 mutants, we measured currents under the conditions with extracellular $\mathrm{pH}$ reduced to $5.5\left(\mathrm{pH} 5 \mathrm{Cl}^{-}\right)$or in the buffer in which $85 \mathrm{mM}$ of chloride was replaced with an equimolar amount of bicarbonate $(\mathrm{pH} 7$ $\left.\mathrm{HCO}^{-}\right)$. The currents were increased by extracellular acidification in $\mathrm{CLH}-1$ mutants as well as wild type (Figure 4-figure supplement 1 and Figure 4-figure supplement 2a-c), indicating that sensitivity to $\mathrm{pH}$ is retained in the mutants. Also, regardless of expressed CLH-1 genotypes, the whole cell currents were comparable between $\mathrm{pH} 7 \mathrm{Cl}^{-}$and $\mathrm{pH}_{7} \mathrm{HCO}_{3}^{-}$conditions as previously reported (Grant et al., 2015, Figure 4-figure supplement 1). These results indicate that under our experimental conditions basic properties of $\mathrm{CLH}-1$ as a voltage-dependent, extracellular $\mathrm{pH}$-sensitive anion channel are retained in CLH-1(M293I) and CLH-1(I146T) mutants.

Next, we asked how clh-1 mutations disturb salt chemotaxis of $C$. elegans. We focused on the salt-sensing neuron ASER, one of the site-of-action of clh-1, and which is essential for food-associated salt concentration chemotaxis (Kunitomo et al., 2013). ASER is activated by salt concentration decreases and deactivated by salt concentration increases (Suzuki et al., 2008, see below). Given that CLH-1 localized to the membranous compartments of ASER and acted as an anion channel, we hypothesized that $\mathrm{CLH}-1$ would be involved in chloride dynamics during salt response of ASER. To 


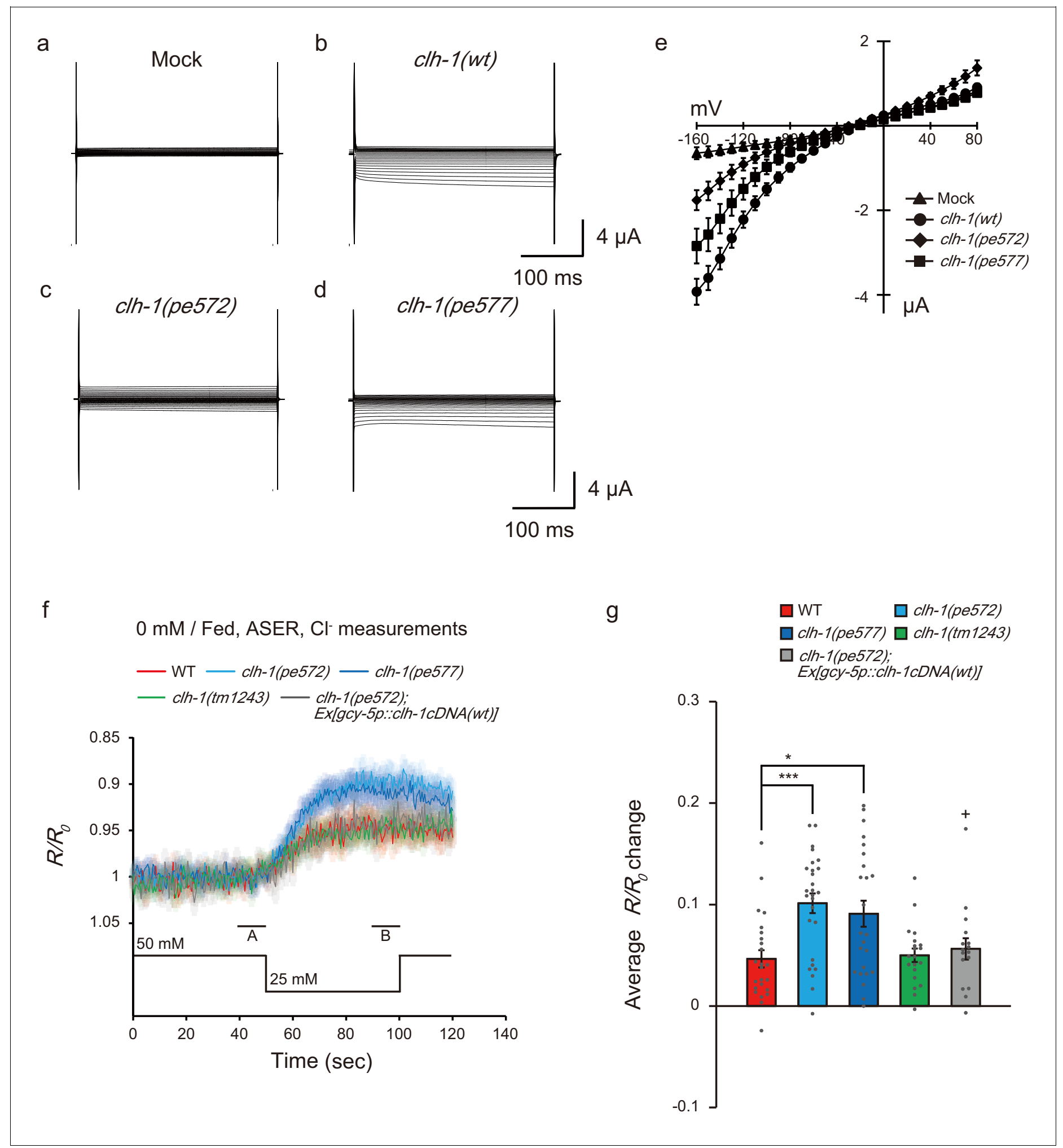

Figure 4. Mutations in clh-1 affect chloride dynamics of ASER in response to salt down-step stimulus. (a-e) Representative current traces from Xenopus oocytes that expressed cRNA for mock (a), clh-1(wt) (b), clh-1(pe572) (c), and clh-1(pe577) (d) clamped at voltages ranging from -160 mV to $80 \mathrm{mV}$ and perfused at $\mathrm{pH} 7$ with $\mathrm{Cl}^{-}$as the dominant extracellular anion. (e) The current-voltage relationships of mock (triangle, $\left.n=7\right), c l h-1(w t)(c i r c l e, n=20), c l h-$ 1(pe572) (diamond, $n=13$ ) and clh-1(pe577) (square, $n=16$ ). The error bars represent s.e.m. (f) Responses of SuperClomeleon expressed in ASER after cultivation at $0 \mathrm{mM} \mathrm{NaCl}$ in the presence of food. External NaCl concentration was shifted from $50 \mathrm{mM}$ to $25 \mathrm{mM}$ at time $50 \mathrm{~s}$. Note that the scale of the vertical axis is inverted so that increase in chloride concentration is displayed as up-shift of traces. A and B indicate the time points for calculating Figure 4 continued on next page 
Figure 4 continued

$R / R_{0}$ changes. The shaded region represents s.e.m., $n \geqq 17$ animals. (g) $R / R_{0}$ changes upon salt decrease. Mean $+/$ - s.e.m., dots represent individual trials. $n \geqq 17$ animals, Tukey's test, ${ }^{* *} p<0.001,{ }^{\star} p<0.05$, compared with wild type. $+p<0.05$, compared with clh-1(pe572) mutant.

The online version of this article includes the following figure supplement(s) for figure 4 :

Figure supplement 1. Extracellular $\mathrm{pH}$-sensitivity and bicarbonate permeability of $\mathrm{CLH}-1$.

Figure supplement 2. Mutant $\mathrm{CLH}-1$ channels retained $\mathrm{pH}$ sensitivity and bicarbonate permeability.

examine this possibility, we monitored intracellular chloride $\left(\left[\mathrm{Cl}^{-}\right]_{\mathrm{i}}\right)$ dynamics of ASER by utilizing the genetically encoded chloride indicator, SuperClomeleon. This probe is a $\mathrm{pH}$-sensitive FRET-type indicator in which YFP/CFP fluorescence ratio decreases upon binding to chloride ions (Arosio and Ratto, 2014; Grimley et al., 2013, see Discussion). Animals were cultivated at $0 \mathrm{mM} \mathrm{NaCl}$ with food and immobilized in a microfluidics device (Chronis et al., 2007), and a $\mathrm{NaCl}$ down-step from $50 \mathrm{mM}$ to $25 \mathrm{mM}$ was applied as salt stimulus. In wild type, the YFP/CFP ratio decreased upon salt downstep, indicating that $\left[\mathrm{Cl}^{-}\right]_{i}$ of ASER was increased when the neuron was activated (Figure 4f). This response is not, at least solely, mediated by CLH-1 because it was also observed in clh-1(tm1243) mutants. Notably, the magnitude of YFP/CFP ratio change was significantly larger in clh-1(pe) mutants, suggesting that ASER $\left[\mathrm{Cl}^{-}\right]_{i}$ greatly increased in the mutants (Figure $4 \mathrm{f}, \mathrm{g}$ ). The enhanced $\left[\mathrm{Cl}^{-}\right]_{i}$ of clh-1(pe572) was restored by expression of clh-1(wt) cDNA in ASER, indicating that CLH-1 acts cell-autonomously in regulation of $\left[\mathrm{Cl}^{-}\right]_{i}$ during depolarization.

\section{ASER salt response is altered in clh-1 mutants}

Intracellular chloride concentration increase can antagonize depolarization of neurons. We then wanted to look into whether clh-1 mutations affect the activity of ASER in response to salt stimulus. As aforementioned, ASER is activated by salt concentration decreases, which is indicated by an increase in intracellular calcium levels, whereas it is deactivated by salt concentration increases (Suzuki et al., 2008). Such ASER responsivity is basically retained regardless of cultivation salt concentrations or food availability (Kunitomo et al., 2013; Oda et al., 2011).

We performed in vivo calcium imaging in wild type and $\mathrm{clh}-1$ mutants using a genetically encoded calcium indicator YC2.6 (Chronis et al., 2007; Horikawa et al., 2010). Animals were cultivated at either $0 \mathrm{mM} \mathrm{NaCl}$ or $100 \mathrm{mM} \mathrm{NaCl}$ with or without food, and ASER was stimulated by repeated salt concentration changes from $50 \mathrm{mM} \mathrm{NaCl}$ to $25 \mathrm{mM} \mathrm{NaCl}$, to observe responses to both down-step and up-step stimuli. After cultivation at $0 \mathrm{mM} \mathrm{NaCl}$, the amplitude of calcium response to the first down-step stimulus was comparable between wild type and clh-1 mutants. However, the response to the second down-step was diminished in clh-1(pe) mutants compared to wild type (Figure 5a,b). A similar trend was observed in the third down-step response. The decay of intracellular calcium level $\left(\left[\mathrm{Ca}^{2+}\right]_{\mathrm{i}}\right)$ was small in the clh-1(pe) mutants, which was more evident after salt up-step (Figure 5-figure supplement $1 a, b)$. This diminished decay was likely responsible for the decreased calcium response to the repeated stimuli. The delayed response of $c / h-1$ (pe572) mutant was rescued by expression of clh-1(wt) cDNA in ASER (Figure 5a,b and Figure 5-figure supplement 1a,b). On the other hand, ASER salt response of clh-1(tm1243) was similar to that of wild type, except that the decay was significantly large during the first down-step stimulus. Interestingly, reduction of ASER response amplitude upon repeated salt down-step stimuli was not obvious after cultivation at 100 $\mathrm{mM} \mathrm{NaCl}$ with food, although clh-1(pe572) constantly showed small ASER responses (Figure $5 c_{\text {, }} d$ and Figure 5-figure supplement $1 c, d)$. Considering the essential role of ASER in salt concentration chemotaxis, these results imply that hampered chemotaxis of clh-1(pe) mutants toward low salt is probably due to the abnormal ASER responsivity to salt concentration changes. We observed expression of a clh-1 promoter-driven transcriptional reporter in ASER and found a reduction of its expression in wild type, but not in the clh-1(pe) mutants, after cultivation at $0 \mathrm{mM} \mathrm{NaCl}$ (Figure 5figure supplement 1e). This result raises a possibility that the expression change of CLH-1 in ASER might contribute to the difference of chloride and calcium responses of the cell between wild type and the clh-1(pe) mutants.

Consistent with the previous reports, salt responses of ASER of starved wild-type animals were not largely different when compared to that of fed animals. However, the activity patterns of clh-1 mutants starved at $0 \mathrm{mM}$ were distinct from those of fed animals (please compare Figure 5, 
a

\section{$0 \mathrm{mM} / \mathrm{Fed}, \mathrm{ASER}, \mathrm{Ca}^{2+}$ measurements}

$$
\begin{aligned}
& -\mathrm{WT}-c / h-1(\text { pe572) }-c / h-1(\text { pe577) } \\
& -c / h-1(\operatorname{tm} 1243)-c / h-1(p e 572) ; \\
& \text { Ex[gcy-5p::clh-1cDNA(wt)] }
\end{aligned}
$$

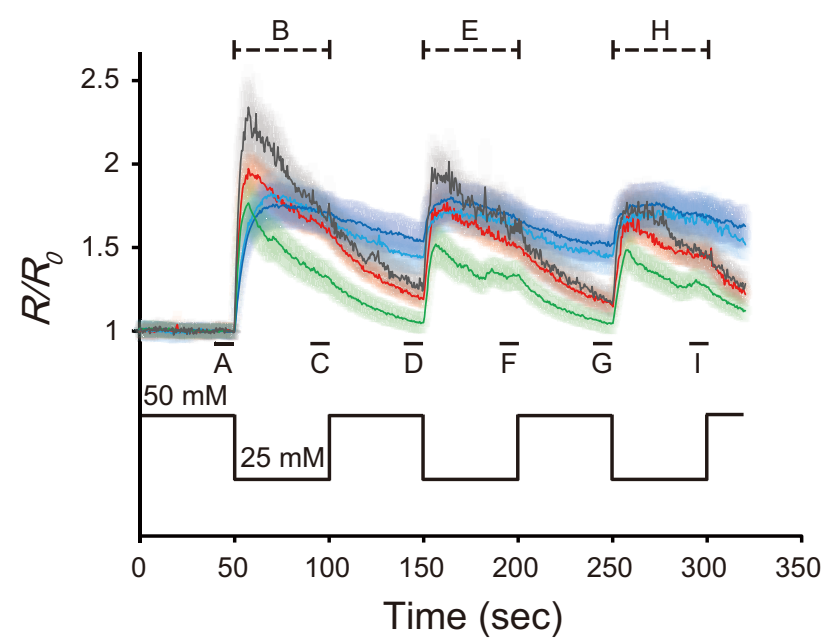

C $100 \mathrm{mM} / \mathrm{Fed}, \mathrm{ASER}, \mathrm{Ca}^{2+}$ measurements
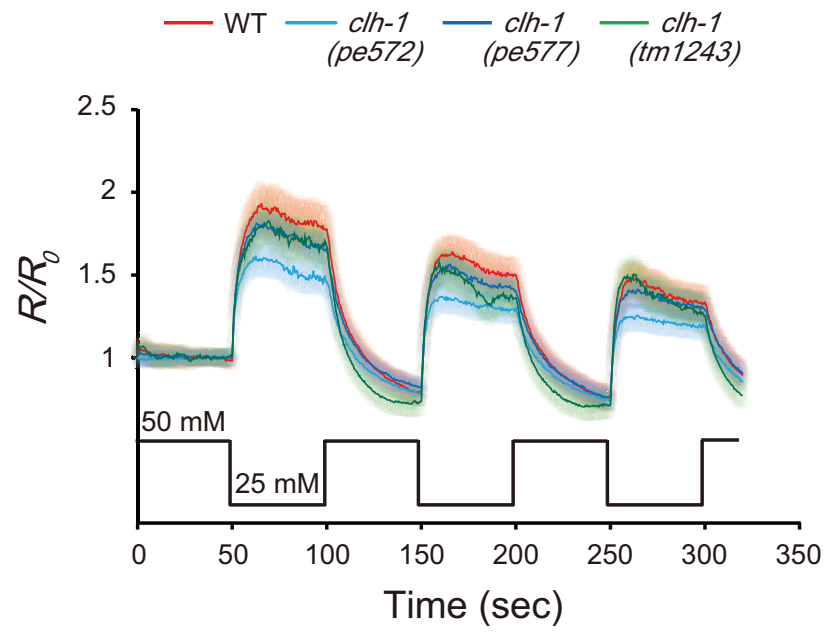

b

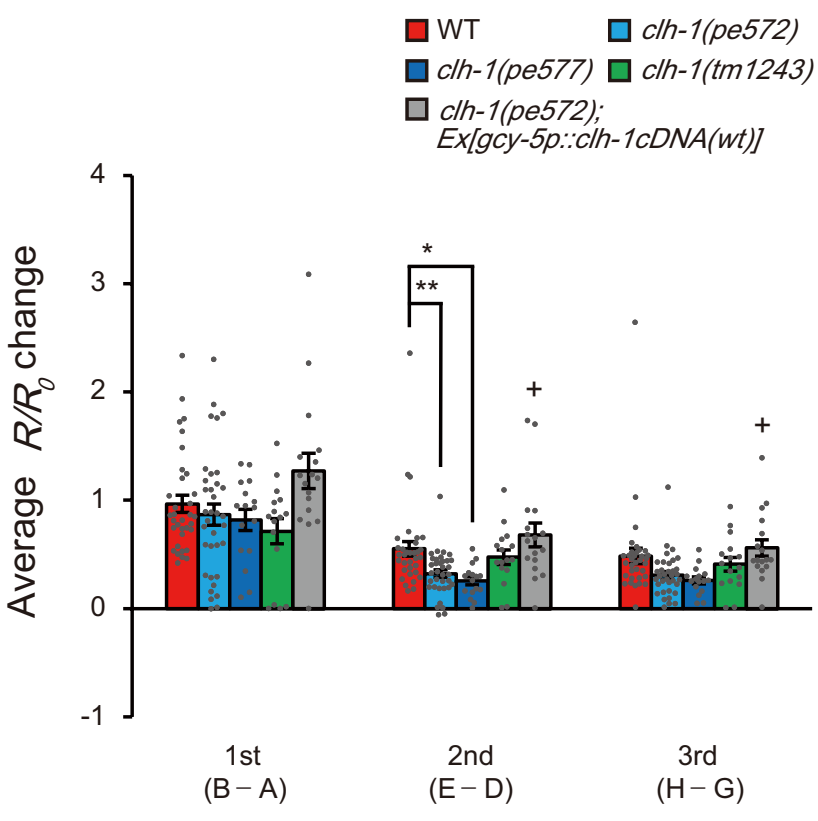

d

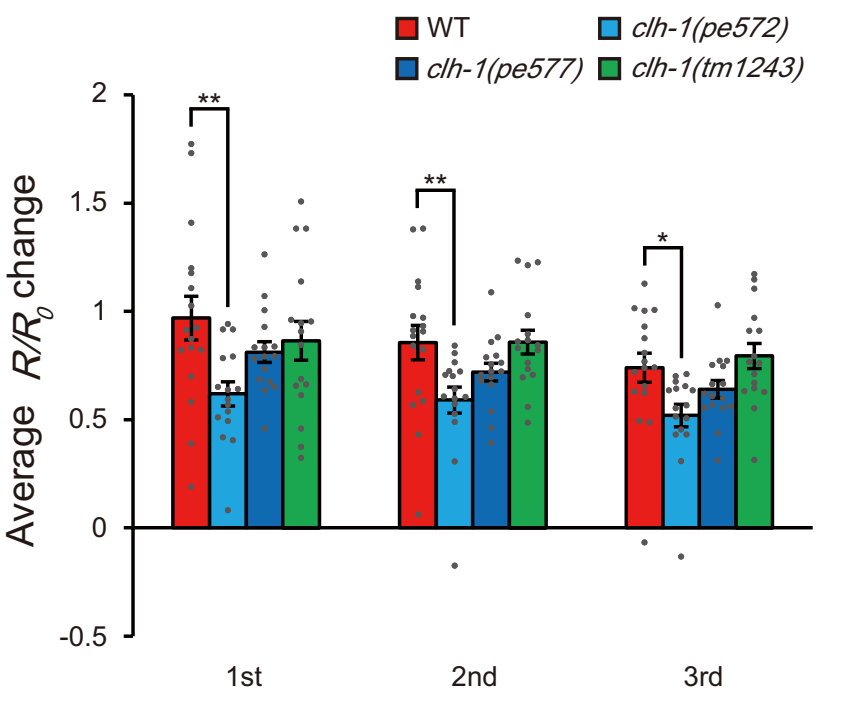

Figure 5. Calcium dynamics of ASER in response to repeated salt stimuli is altered in clh-1 mutants. (a and c) Calcium responses of ASER stimulated by $\mathrm{NaCl}$ concentration changes between $50 \mathrm{mM}$ and $25 \mathrm{mM}$ after cultivation at $0 \mathrm{mM} \mathrm{NaCl}$ (a) or $100 \mathrm{mM} \mathrm{NaCl}$ (c) in the presence of food for 6 hr. A to I indicate the time points for calculation of $R / R_{0}$ changes. $A, D$, and $G$ are the time points for pre-stimulus $R / R_{0}, B, E$, and $H$ are the time points for peak $R / R_{0}$ during stimulation, $C, F$, and I are the time points for decayed $R / R_{0}$ during stimulation. The shaded region represents s.e.m., $n \geqq 16$ animals. (b and d) $R / R_{0}$ changes at each $\mathrm{NaCl}$ down-step stimulus ( $\mathrm{B}-\mathrm{A}, \mathrm{E}-\mathrm{D}$, and $\mathrm{H}$ - G for the 1st, 2nd, and 3rd stimulus, respectively). 0 mM NaCl cultivated (b) or $100 \mathrm{mM}$ cultivated (d). See Materials and methods for details. Bars and the error bars represent mean $+/$ - s.e.m., dots represent individual trials. $n \geqq$ 16 animals, Tukey's test, ${ }^{* \star} p<0.01,{ }^{*} p<0.05 .+p<0.05$, compared with clh-1(pe572) mutant.

The online version of this article includes the following figure supplement(s) for figure 5 :

Figure 5 continued on next page 
Figure 5 continued

Figure supplement 1. clh-1 missense mutations affect calcium dynamics of ASER.

Figure supplement 2. Effect of clh-1(pe) mutations on calcium dynamics of ASER in starved animals.

Figure 5-figure supplement 1 and Figure 5-figure supplement 2). After cultivation at $0 \mathrm{mM}$ without food, for example, the amplitude of activation of clh-1(pe577) mutants were even larger than that of wild type (Figure 5-figure supplement 2a-c). Meanwhile, the difference of ASER salt responses between wild type and clh-1(pe) mutants were less obvious after starvation at $100 \mathrm{mM}$ $\mathrm{NaCl}$ (Figure 5-figure supplement 2e-h). Taking all these into account, we concluded that mutations in clh-1 affect responsivity of ASER, most notably in the reduced response to repeated salt down-step and up-step stimuli after cultivation at $0 \mathrm{mM} \mathrm{NaCl}$ with food.

\section{Behavioral strategies for chemotaxis are disrupted in clh-1(pe) mutants}

Next, we quantitatively analyzed the navigation behavior of clh-1 mutants to examine which behavioral components are affected. C. elegans utilize at least two behavioral strategies to achieve salt chemotaxis: klinokinesis and klinotaxis. In klinokinesis, migration bias is generated by controlling the frequency of steep turns called pirouettes, which are typically accompanied by reversals and omega turns. The bout of pirouette is triggered according to cumulative salt concentration change along an animal's progression (Pierce-Shimomura et al., 2001). In klinotaxis, animals gradually curve toward their preferred direction by sensing fluctuation of salt concentration accompanying head bending (lino and Yoshida, 2009). Input to ASER is both required and sufficient for fed animals to generate the two behavioral strategies (Kunitomo et al., 2013; Satoh et al., 2014). We found that klinotaxis bias was severely impaired in clh-1(pe) mutants regardless of cultivation salt concentrations (Figure 6a and Figure 6-figure supplement 1a,c). Klinokinesis bias of the clh-1(pe) animals after cultivation at $100 \mathrm{mM} \mathrm{NaCl}$ was comparable to that of wild type (Figure $\mathbf{6} \mathbf{b}$ and Figure 6-figure supplement $1 \mathrm{~d}$ ). These results suggest that chemotaxis of $\mathrm{clh}-1$ (pe) animals to high salt after cultivation at $100 \mathrm{mM} \mathrm{NaCl}$ largely depends on klinokinesis. However, after cultivation at $0 \mathrm{mM} \mathrm{NaCl}, \mathrm{klino-}$ kinesis bias was lost in clh-1(pe) mutants. Up-regulation of pirouette frequency along with salt concentration increase $(d C / d t>0)$ was abolished in $c / h-1(p e)$ mutant animals (Figure $6 \mathrm{~b}$ and Figure 6-figure supplement $1 \mathrm{~b}$ ). These results strongly indicate that the defective chemotaxis of clh-1 (pe) mutants is due to loss of both klinotaxis and klinokinesis biases after cultivation at $0 \mathrm{mM} \mathrm{NaCl}$. The klinokinesis and klinotaxis of clh-1(tm1243) mutants were comparable to those of wild type (Figure $6 a, b$ and Figure 6-figure supplement $1 a, b)$.

\section{Reduced salt response of AIB to salt increase in clh-1(pe572) mutants}

To further gain insight into the neural mechanism of klinokinesis defects of the clh-1(pe) mutants after cultivation at $0 \mathrm{mM} \mathrm{NaCl}$, we focused on AIB, a postsynaptic interneuron of ASER which promotes sensory stimulus-dependent reversals, the trigger of pirouettes (Piggott et alı, 2011; Zou et al., 2018). Because the synapse between ASE and AIB is proposed as the site of plasticity that regulate klinokinesis bias in salt chemotaxis (Kunitomo et alı, 2013; Luo et al., 2014; Wang et al., 2017), we hypothesized that the responsivity of AIB may be altered in these mutants. To examine this possibility, we observed salt responses of AIB in freely behaving animals using a microfluidics arena (Albrecht and Bargmann, 2011). Animals were cultivated at $0 \mathrm{mM} \mathrm{NaCl}$ with food and stimulated by an up-step $\mathrm{NaCl}$ stimulus from $0 \mathrm{mM}$ to $25 \mathrm{mM}$. Wild-type animals responded to the salt stimulus by slowing down or reversal (the moving velocity becomes less than zero, Figure $6 c$ and Figure 6-figure supplement 2). clh-1(pe572) mutants also showed reduction in speed, but the proportion of animals that exhibited reversal was smaller than wild type (Figure 6d). These results agreed with the klinokinesis defect observed on chemotaxis plates (Figure $6 \mathrm{~b}$ and Figure 6-figure supplement $1 \mathrm{~b}$ ). Importantly, responses of AIB to salt up-step correlated well with behaviors. AIB was largely activated upon salt stimulus in wild type, which was absent in clh-1(pe572) mutants (Figure 6e,f). Expression of clh-1(wt) cDNA in ASER restored both reversal behavior and AIB response, indicating that malfunction of clh-1 in ASER caused the defects. Collectively, our results indicate that impaired salt responses of ASER-AIB salt circuit resulted in 


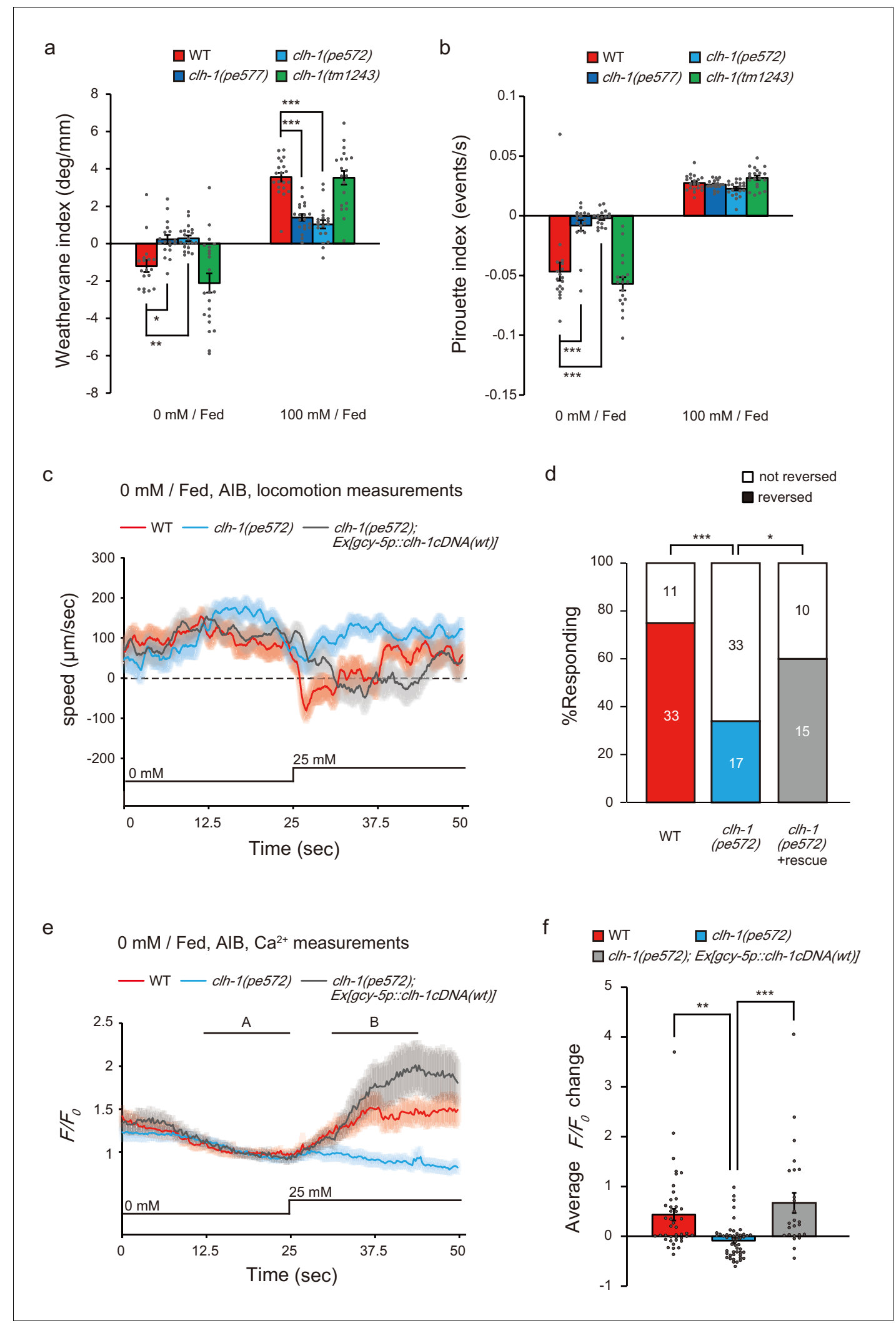

Figure 6. Missense mutations in clh-1 attenuate both klinotaxis and klinokinesis, as well as AIB response and reversal in response to salt increase. (a and b) clh-1(pe) mutants show defects in migration bias in salt chemotaxis. Bias of klinotaxis (a) and klinokinesis (b), represented by weathervane index and pirouette index, respectively. In both mechanisms, positive and negative values indicate migration bias toward higher and lower salt concentrations, respectively. Bars and the error bars represent mean +/- s.e.m., dots represent individual trials. $n \geqq 18$ assays, Dunnett's test, Figure 6 continued on next page 
Figure 6 continued

${ }^{* * \star} \mathrm{p}<0.001,{ }^{* \star} \mathrm{p}<0.01,{ }^{*} \mathrm{p}<0.05$. (c and e) Locomotion speed of animals (c) and calcium responses of AlB (e) after cultivation at $0 \mathrm{mM} \mathrm{NaCl}$ in the presence of food. In panel (e), A and B indicate the time points for calculation of $F / F_{0}$ changes. $\mathrm{NaCl}$ concentration change from $0 \mathrm{mM}$ to $25 \mathrm{mM}$ at 25 s. The shaded region represents s.e.m., $n \geqq 25$ animals. (d) Proportion of animals that showed reversal after salt stimulus. Reversal was defined as follows; backward locomotion, whose velocity less than $-100 \mu \mathrm{m} / \mathrm{sec}$ was continued for more than $1 \mathrm{~s}$ ( 35 frames). The error bars represent s.e.m., $\mathrm{n} \geqq$ 25 animals, Fisher's exact test. ${ }^{\star \star *} \mathrm{p}<0.001,{ }^{\star} \mathrm{p}<0.05$. (f) $\mathrm{F} / \mathrm{F}_{0}$ change upon salt stimulus (B - A, see Materials and methods for details). Bars and the error bars represent mean + /- s.e.m., dots represent individual trials. $n \geqq 25$ animals, Tukey's test, ${ }^{\star \star *} p<0.001,{ }^{\star *} p<0.01$.

The online version of this article includes the following figure supplement(s) for figure 6 :

Figure supplement 1. Quantification of the navigation behaviors.

Figure supplement 2. Quantification of reversal behaviors in microfluidics arena.

reduction of turning frequency in clh-1(pe572) mutants upon salt up-step after cultivation at $0 \mathrm{mM}$ $\mathrm{NaCl}$.

\section{Discussion}

Here, using genetic, neurophysiological, and behavioral analyses, we described a neural mechanism in which novel missense mutations of the $\mathrm{ClC}$ chloride channel clh-1 disturb responses of a sensory neuron and eventually alter animal's behavior in C. elegans. This is probably carried out by impairment of, (i) ASER responsivity to repeated salt stimuli that affect klinotaxis and (ii) salt up-step response of ASER and thereby of AIB after cultivation at low salt that contribute to klinokinesis (Figure 7). Repeated activation of ASER synchronized with head swing generates biased klinotaxis (Satoh et al., 2014). Reduced responsivity to repeated salt stimuli in clh-1(pe) mutants implied that temporal resolution of ASER is impaired in the mutants (Figure 5a-b and Figure 5-figure supplement 1a). Klinotaxis was actually disrupted in the mutants regardless of previous cultivation conditions (Figure 6a and Figure 6-figure supplement 1a,c). Our data is consistent with the idea that dynamic $\left[\mathrm{Ca}^{2+}\right]_{i}$ fluctuation in ASER reflecting environmental $\mathrm{NaCl}$ concentration change is required for generation of klinotaxis. In addition, clh-1(pe) mutants showed klinokinesis defect upon salt increase after cultivation at $0 \mathrm{mM} \mathrm{NaCl}$ (Figure $6 \mathrm{~b}$ and Figure 6 -figure supplement $1 \mathrm{~b}$ ). In agreement with this, responses of ASER and AIB, and reversal behavior upon salt up-step were reduced in clh-1(pe572) animals (Figure 5a and Figure $6 \mathrm{~d}, \mathrm{f}$ ). Suppression of AIB activity results in reduction of turning frequency (Gordus et al., 2015; Piggott et al., 2011). Unresponsiveness of AIB to salt increase will therefore result in reduced migration toward low salt regions. Defects in migration to low salt can explain the increased salt preference of the clh-1(pe) mutants after cultivation at $50 \mathrm{mM}$ $\mathrm{NaCl}$ (Figure 1b). Wild-type animals wander around the central area on chemotaxis assay plate by suppressing migration toward both lower and higher salt concentrations after cultivation at $50 \mathrm{mM}$ $\mathrm{NaCl}$ (Kunitomo et al., 2013; Ohno et al., 2014). Therefore, the loss of migration bias toward low salt under such condition would drive animals toward high salt.

Monitoring of $\left[\mathrm{Cl}^{-}\right]_{i}$ using SuperClomeleon indicated an influx of chloride into ASER upon activation of the cell. In general, influx of anion prevents depolarization in neurons (Staley et al., 1995). There are several possible mechanisms that could explain increased chloride influx by clh-1(pe) mutations (Figure 7, middle). One is an elevated anion intrusion via CLH-1(pe) channels. CIC-2, the closest mammalian homolog of $\mathrm{CLH}-1$, was shown to be involved in chloride influx into neuronal cells, probably due to incomplete rectification (Ratté and Prescott, 2011; Rinke et al., 2010). Another possible mechanism is a difference in ASER's transmembrane chloride potential between genotypes. $\mathrm{ClC}$ proteins, as well as other chloride transporters such as $\mathrm{K}^{+} / \mathrm{Cl}^{-}$cotransporters (KCCs) and $\mathrm{Na}^{+} /$ $\mathrm{K}^{+} / \mathrm{Cl}^{-}$cotransporters (NKCCs), are involved in the excitability of neurons through their homeostatic roles in regulating cellular ionic milieu (Jentsch, 2008; Stauber et al., 2012). It remains unclear how extracellular chloride of ASER is regulated. In this study, we showed that clh-1(wt) cDNA failed to rescue clh-1(pe572) phenotype if expressed in AmSh or ciliated neurons together with ASER (Figure $3 b$ ). This result suggests that $\mathrm{CLH}-1$ may differently act depending on cell type. Indeed, expression of a $\mathrm{K}^{+} / \mathrm{Cl}^{-}$cotransporter $\mathrm{KCC}-3$ is restricted in glial cells including AmSh (Katz et al., 2019; Spencer et al., 2011; Wallace et al., 2016). KCC-3 is involved in responsivity of the thermosensory neuron AFD through regulating extracellular chloride level of the sensory endings (Singhvi et al., 2016; Yoshida et al., 2016). Since chloride milieu of a cell is maintained by a balance 


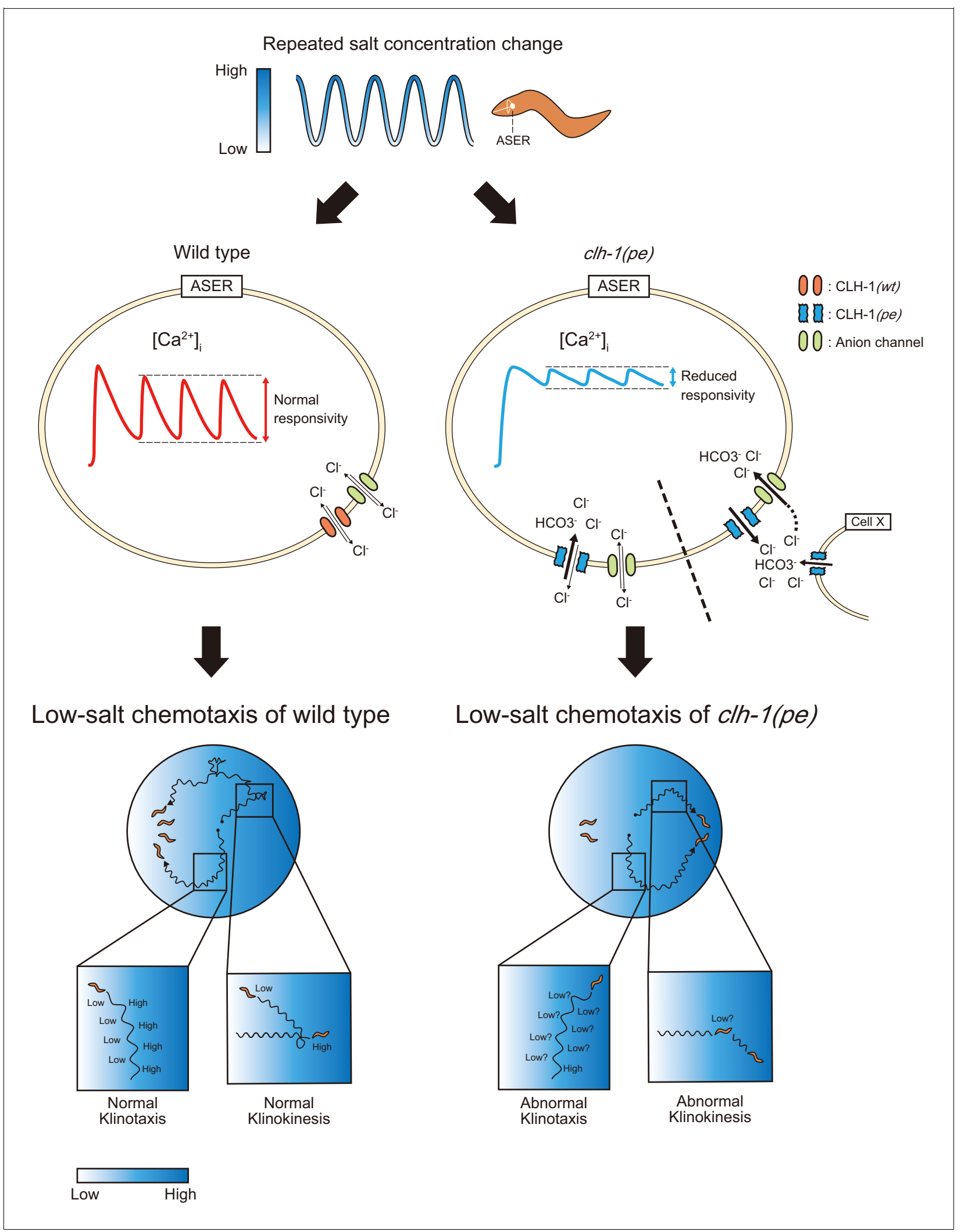

Figure 7. A model for the mechanism of impaired low salt chemotaxis of clh-1(pe) mutants. Animals experience salt concentration fluctuation along with migration on salt gradient, which is depicted by blue waves (top). Salt information is sensed by the ASER neuron and translated into intracellular calcium transients (middle). In the clh-1(pe) mutants, calcium responses of ASER to repeated salt stimuli is diminished after cultivation at low-salt concentration. This is probably due to increased influx of chloride ions into the cell upon depolarization. Permeation of bicarbonate ions through $\mathrm{CLH}-1$ Figure 7 continued on next page 
Figure 7 continued

may also be involved. Although the mechanism for increased chloride influx is currently unknown, we suggest two possible scenarios; increased anion influx through CLH-1(pe) channels (left of the dashed line) and elevated extracellular chloride level (right of the dashed line). Reduced responsivity of ASER then results in hampered migration bias toward low salt both in klinokinesis and klinotaxis (bottom).

of these channels and transporters activity, the aforementioned chloride regulation could be disturbed when $\mathrm{CLH}-1$ was chronically overexpressed in AmSh.

SuperClomeleon is an FRET-based sensor sensitive to pH change (Arosio and Ratto, 2014; Grimley et al., 2013). In addition, activity-dependent cytoplasmic acidification is reported in C. elegans sensory neurons (Ventimiglia and Bargmann, 2017). As we observed the SuperClomeleon responses upon activation of ASER, there is a possibility that cytoplasmic $\mathrm{pH}$ change confounded the measurement of chloride concentration. Furthermore, $\mathrm{CLH}-1$ is $\mathrm{HCO}_{3}^{-}$-permeable and contributes to cellular $\mathrm{pH}$ regulation (Grant et al., 2015). Hence, $\mathrm{pH}$ regulation might be altered in clh-1 (pe) mutants in vivo (Figure 7, middle right). These scenarios, together with further characterization of channel properties of $\mathrm{CLH}-1$, needs to be demonstrated in the future study.

In the levamisole resistance test, missense (pe572 and pe577) and the deletion (tm1243) allele of clh-1 showed an opposite phenotype (Figure 1-figure supplement 3c). In addition, overexpression of clh-1(wt) impaired low-salt chemotaxis, which partially resembled the phenotype of clh-1(pe) mutants (Figure 1c). These results suggest that the clh-1(pe) mutations are neomorphic or perhaps hypermorphic alleles. CIC proteins act in homo- or heterodimers (Stölting et al., 2014). It is not predictable whether $\mathrm{CLH}-1$ (pe)/CLH-1(wt) heterodimer shows mutant-type or wild-type activity. The recessiveness of $\mathrm{clh}-1$ (pe) mutations (Figure 1d) can be explained by assuming that the heterodimer shows wild-type activity. Although genetic analyses indicated that the mutations of clh-1 affected the property of $\mathrm{CLH}-1$ channels, we did not find a remarkable change in the electrophysiological analyses except that currents were smaller in the mutants. Limitations of using heterologous system may explain such disagreement (reviewed in Bianchi, 2006; Dascal, 2000). First, cellular environment of Xenopus oocyte differs from that of $C$. elegans neurons. Inward currents were observed in membrane potential range as low as $-80 \mathrm{mV}$ or lower in electrophysiology, whereas responses of ASER was observed under depolarized conditions (Shindou et alo, 2019). In addition, modification of $\mathrm{CLH}-1$ proteins or interaction of $\mathrm{CLH}-1$ with other proteins may be absent in Xenopus oocyte. The CBS domain is known to regulate channel activity depending on phosphorylation or binding to auxiliary subunits (Denton et al., 2005; Estévez et al., 2004; Stölting et alo, 2013). Such modifications may also occur in CLH-1. Regarding the amplitude of whole-cell membrane current, the expression level of channel proteins might have been different between wild type and mutants. Stability of channel proteins also could be affected by the mutations. To date, substantial number of mutations have been characterized in $\mathrm{ClC}$ genes, most of which are mammalian $\mathrm{ClCs}$. To our knowledge, however, none of these mutations were similar to $\mathrm{clh}-1(\mathrm{pe})$ mutations. Future studies such as single-channel analyses are required to fully characterize the property of mutant $\mathrm{CLH}-1$ channels.

ASER makes synapses to three first layer interneurons, AIA, AIB and AIY. Activation of AIA and AIY are known to promote forward locomotion, while activation of AIB promotes reversals (Gray et al., 2005; Piggott et al., 2011; Zou et al., 2018). The salt response of AIB is markedly changed by salt experience. AIB is activated by salt concentration decrease if previously-experienced salt concentrations were high, whereas it is rather deactivated after cultivation at low salt (Kunitomo et al., 2013; Luo et al., 2014). Here, we showed that AIB is activated by salt increase after cultivation at low salt, which was reduced in clh-1(pe572) mutant (Figure 6e,f). This response is at least partially dependent on ASER because AIB response was restored by expression of clh-1(wt) in ASER. Considering that ASER is glutamatergic (Serrano-Saiz et al., 2013), there may be both excitatory and inhibitory transmission between ASER and AIB. Indeed, AIB expresses the AMPAtype glutamate receptor GLR-1, which mediates excitatory glutamatergic inputs from sensory neurons (Chalasani et al., 2007; Zou et al., 2018). Besides, it has recently been suggested that the glutamate-gated chloride channel GLC-3 and AVR-14 would mediate inhibitory inputs to AIB (Kuramochi and Doi, 2018; Summers et al., 2015). These observations raise the possibility that glutamate response of AIB depends on the electrochemical gradient of $\mathrm{Cl}^{-}$across the membrane as well as the balance of excitatory and inhibitory receptors. In other words, under the condition in which inhibitory glutamate receptors were dominant, AIB could be disinhibited by reduction of 
presynaptic glutamate release. Growing evidence highlights the importance of chloride homeostasis in the function of the nervous system. In mammalian hippocampal neurons, intracellular chloride is elevated during embryonic development and thereby renders GABAergic transmission excitatory, which is necessary for maturation of synaptic network (Pfeffer et al., 2009). Furthermore, chloride transportation through NKCC1 regulates synaptic plasticity and memory formation in adult hippocampal neurons (Deidda et al., 2015). It will be of interest in the future to determine how the extracellular ionic milieu and glutamate receptors orchestrate the responsiveness of AIB.

In this study, we showed that $\mathrm{ClC}$ channel/transporter genes function redundantly in salt chemotaxis of C. elegans. Food-associated salt chemotaxis was normal in each single (clh-1(tm1243), clh-2 (ok636), clh-3(ok763), or clh-4(ok1162)) or clh-2(ok636) clh-1(tm1243) double mutants. However, lack of three ClC channels, clh-3(ok763) clh-2(ok636) clh-1(tm1243) triple mutation, gave a marked effect on behavior (Figure 2-figure supplement $1 \mathrm{~b}$ and Figure $2 \mathrm{~b}$ ). Interestingly, these three genes are located very closely on chromosome II $(4.08+/-0.003 \mathrm{cM}$ for clh-1, 3.46 +/- $0.003 \mathrm{cM}$ for clh-2, 0.50 $+/-0.000 \mathrm{cM}$ for $\mathrm{clh}-3)$, suggesting that they are derived by duplication and they might share some evolutionarily conserved functions. The clh-5 putative anion transporter gene is also located on chromosome II: $1.01+/-0.007 \mathrm{cM}$. clh-5(tm6008) and many of the clh multiple mutants that carry clh-5 (tm6008) showed high immobility indices (Figure 2-figure supplement 1b,c and Figure 2-figure supplement 1a), suggesting that the mutants displayed a locomotion defect on chemotaxis assay plate. Locomotion defects can interfere with chemotaxis of the animals since it prevents prompt migration toward preferred conditions. Thus, improvement of locomotion might ameliorate the chemotaxis defects in these mutants. Actually, mutation of clh-3 partially suppressed chemotaxis defect of the clh-5 clh-2 clh-1 triple mutation (that is, in the clh-5 clh-3 clh-2 clh-1 quadruple mutants) as well as locomotion defects. These results imply that $c / h-5$ and $c / h-3$ are involved in locomotion upon chemotaxis. Mutation of clh-6(tm617) V, which encodes a putative anion transporter and whose single mutation had no effect on salt chemotaxis, also gave rise to salt chemotaxis defect in combination with clh-2(ok636) clh-1(tm1243) II mutations.

As we did not examine all combinations of multiple loss-of-function mutation but started with the clh-2(ok636) clh-1(tm1243) double mutant, it is unclear whether clh-1 is naturally involved in salt chemotaxis. Although loss of clh-1 showed no remarkable salt chemotaxis defects, it affected salt responses of ASER under starvation conditions (Figure 5-figure supplement 2). Function of the sensory neurons other than ASER are required to properly avoid the salt concentration of starved conditions (Jang et al., 2019; Watteyne et al., 2020). Cooperation of multiple sensory neurons might have compensated for the defective salt response of ASER. Intriguingly, animals that lacked all $\mathrm{CIC}$ genes were viable, but showed severe salt chemotaxis defect. Only a few studies so far have addressed functional redundancy of ClC family proteins in an organism (Jeworutzki et al., 2014; Stölting et al., 2014), and our study provides an insight into functional differences and redundancies of $\mathrm{ClC}$ family proteins.

\section{Materials and methods}

\section{C. elegans strains and culture}

Bristol N2 was used as wild-type $C$. elegans. All mutant strains were outcrossed multiple times with N2. E. coli NA22 was used as a food source for behavioral analyses including salt chemotaxis assay. For imaging experiments, OP50 was used as a food source. Strains used in this study are listed in Supplementary file 1.

\section{Behavioral tests}

Salt chemotaxis assays were performed as previously reported with minor modifications (Kunitomo et al., 2013). Chemotaxis assay plate was prepared as follows. On top of an agar plate (2\% agar, $25 \mathrm{mM}$ potassium phosphate $(\mathrm{pH}$ 6.0), $1 \mathrm{mM} \mathrm{CaCl} 2,1 \mathrm{mM} \mathrm{MgSO}, 8.5 \mathrm{~cm}$ in diameter and $1.76 \mathrm{~mm}$ in thickness with $10 \mathrm{~mL}$ agar), $\mathrm{NaCl}$ gradient was created by placing two cylindrical agar blocks (14.5 mm in diameter and $5.3 \mathrm{~mm}$ in thickness) that contained $0 \mathrm{mM}$ (position A) or $150 \mathrm{mM}$ (position B) of $\mathrm{NaCl}$ in the composition of background plate. The agar blocks were removed after 18 to $20 \mathrm{hr}$, just before assay. Animals were cultivated on regular nematode growth medium (Brenner, 1974) to young adults, and further cultivated on NGM plates that contained either $0 \mathrm{mM}$ or 
$100 \mathrm{mM} \mathrm{NaCl}$ in the presence or absence of food for pre-assay cultivation for $6 \mathrm{hr}$. Fifty to 200 worms were collected, washed twice with wash buffer $(50 \mathrm{mM} \mathrm{NaCl}, 25 \mathrm{mM}$ potassium phosphate ( $\mathrm{pH}$ 6.0), $1 \mathrm{mM} \mathrm{CaCl}_{2}, 1 \mathrm{mM} \mathrm{MgSO}_{4}, 0.02 \%$ gelatin), then placed at the center of the assay plate. Animals were allowed to run for $45 \mathrm{~min}$. One microliter each of $0.5 \mathrm{M} \mathrm{NaN}_{3}$ was spotted to the position $A$ and $B$ so that worms that had reached to these positions were immobilized. The number of worms $(N)$ within area $A$ and $B$ (a $2 \mathrm{~cm}$ radius from the center of each agar cylinder's position $A$ and $B$ ) and area $O$ (ellipse with radii $20 \mathrm{~mm}$ and $10 \mathrm{~mm}$ around the start point) as well as the total number of worms on assay plate were counted to calculate the chemotaxis index and immobility index as follows (Figure 1-Figure 1-figure supplement 1a).

$$
\begin{aligned}
& \text { Chemotaxis index }=\left(N_{B}-N_{A}\right) /\left(N_{\text {Total }}-N_{O}\right) \\
& \text { Immobility index }=N_{O}-N_{\text {Total }}
\end{aligned}
$$

The values of chemotaxis index 1.0 and -1.0 represent complete migration toward high and low salt regions, respectively, whereas zero value represents no preference for salt concentration (unbiased migration) or a preference for concentration near the central region. Levamisole resistance was determined on agar plates that contained $0.5 \mathrm{mM}$ drug at room temperature. Body paralysis was defined as the lack of body movement in response to prodding by toothpick by visual inspection every 15 min (Gottschalk et al., 2005; Lewis et al., 1980).

\section{Forward genetic screening and identification of the responsible gene}

Wild-type animals were mutagenized with ethyl methanesulfonate (EMS) as described (Brenner, 1974). Progenies were tested for food-associated salt concentration chemotaxis and mutants were selected; animals that showed defective chemotaxis were collected from the assay plate and propagated for testing at the next generation. For detail, we collected animals that approached high-salt region (the region $B$ in Figure 1-figure supplement 1a) after cultivation at $0 \mathrm{mM} \mathrm{NaCl}$ (Figure 1-figure supplement 1b). This strategy could yield two types of mutant candidates; ones that preferred high-salt concentration and ones that show unbiased preference to salt concentration. The latter tend to randomly distribute on salt gradient and therefore could come to high-salt region by chance. JN572 and JN577 showed an unbiased salt chemotaxis after cultivation at 0 mM with food, but they showed an obvious preference to high salt after cultivation at $50 \mathrm{mM}$ or higher, demonstrating that the mutants are intrinsically capable of chemotaxis. This process was repeated until F6. We screened approximately 24,000 F2 animals and obtained seven independent mutants. After being outcrossed with N2, two mutant strains JN572 and JN577 were further analyzed. Other mutants will be described elsewhere.

We mapped the responsible gene for JN572 and JN577, each respectively appeared to be clh-1 (pe572) and clh-1(pe577) (see text), by using single nucleotide polymorphisms (SNPs) between N2 and CB4856 (Fay and Bender, 2006; Wicks et al., 2001) Both of these mutations were mapped between 2.82 (SNP: WBVar00175127) and 6.12 (SNP: WBVar00176673) on chromosome II. Genome sequences identified a missense mutation in clh-1 in each strain. We performed rescue experiments using fosmids and genomic PCR fragment of candidate genes. Both mutants were successfully rescued by WRM0612bF09 (fosmid) or genomic PCR fragment of clh-1/T27D12.2. Thus we concluded clh- 1 was the causative gene. pe 572 was a $G$ to $A$ transition, whose $5^{\prime}$ and $3^{\prime}$ flanking sequences are TGCACATTCTCGGCGCCTAT and GGAGGTAGGGCTTAACCCTT, respectively. pe577 was a $T$ to C transition, whose $5^{\prime}$ and $3^{\prime}$ flanking sequences are GATTTTCATCGATATGGGAA and TGAGTATC TGATTCATTGTG, respectively.

\section{Genotyping}

Alleles of each gene locus were verified by PCR using sequence-specific primers for the target sequences. Genotyping primers used in this study are listed in Supplementary file 2.

\section{clh-1 expression constructs}

Full length clh-1a/T27D12.2a cDNA clones for wild type, clh-1(pe572) and clh-1(pe577) mutants were obtained by RT-PCR using sequence-specific primers (5'- GCTAGCCAGGATGGAAGACGCCG TCGTCGT $-3^{\prime}$ and 5'- GGTACCTTAGCGGGTTTCGTCATCCG $-3^{\prime}$ ). PCR products were cloned to the pDEST vector (Invitrogen) as a Nhel-Kpnl fragment, and whose sequence was confirmed. 
clh-1 genomic DNA fragments (clh-1gDNAs) were prepared by PCR using sequence-specific primers (5'- ATTGCACACATAATTGCGGTAGAC -3' and 5'- TTGACCCATAAGGTGTAGGCTGC - 3'). The nucleotide sequence of the open-reading frame was confirmed after cloning.

\section{Plasmid construction}

Vectors for cell-specific expression in C. elegans were generated using the Gateway cloning system (Invitrogen). For clh-1 promoter, a $7.5 \mathrm{~kb}$ DNA fragment that contained $4.0 \mathrm{~kb}$ upstream of transcription initiation site and $3.5 \mathrm{~kb}$ downstream of the first exon of clh-1a were amplified from $C$. elegans genome using primers: 5'- GCACACATAATTGCGGTAGAC $-3^{\prime}$ and 5'- CGCATTTTCTTGAACCC TGG $-3^{\prime}$ and cloned into an entry vector, pENTR-1A. For vap-1 promoter, which specifically expresses in amphid sheath glial cells (Bacaj et al., 2008), we amplified $2.5 \mathrm{~kb}$ upstream of the first exon of vap-1 using primers: 5'- ATTTATAGAAAGTTTCCAAA $-3^{\prime}$ and 5'- CTGTGAAAATGAACGCACGC - 3'. pDEST-SL2::nls4::mTFP was generated by ligating the trans-spliced leader sequence $\mathrm{SL2}$, four repeats of the nuclear localization signal, and the fluorescence protein $\mathrm{mTFP}$ on a pDEST vector. For pDEST-clh-1cDNA::mTFPs, Kpnl-EcoRV fragment from pDEST-mTFP was cloned into the pDEST-clh-1cDNA vectors. SuperClomeleon (Grimley et al., 2013) was codon-optimized for expression in C. elegans using Codon adapter (Redemann et al., 2011), synthesized, and cloned into pDEST vector. The expression constructs pG-clh-1p::nls4::mTFP, pG-rimb-1p::clh-1(wt)cDNA, pGgcy-5p::clh-1(wt)cDNA, pG-gcy-7p::clh-1(wt)cDNA, pG-dyf-11p::clh-1(wt)cDNA, pG-vap-1p::clh-1(wt) cDNA, pG-gcy-5p::SuperClomeleon were created by site-specific recombination between pENTR and PDEST plasmids. Information of all plasmids used in this study can be found in Supplementary file 3 (list and use) and Supplementary file 4 (nucleotide sequences).

\section{Generation of transgenic animals}

Expression constructs or genomic PCR fragments were injected at $0.1-50 \mathrm{ng} / \mu \mathrm{l}$ along with a coinjection marker (in many case pG-myo-3p::venus or pG-lin-44p::GFP, 5-10 ng/ $\mu$ ) and pPD49.26 (a gift from Andrew Fire, up to $100 \mathrm{ng} / \mu \mathrm{l}$ ) as a carrier DNA. For comparison among genotypes, the transgene was initially introduced into wild-type background and transferred to other genetic backgrounds by cross. JN2249 was generated by injecting pG-clh-1p::nls4::mTFP and lin-44p::mCherry into an expression marker strain Is[rimb-1p::nls4::mCherry; eat-4p::nls4::tagRFP; lin-44p::GFP].

\section{Fluorescence microscopy and measurement of sensory cilium length}

Day one adults were mounted on $5 \%$ agar and anesthetized by $10 \mathrm{mM} \mathrm{NaN}_{3}$, or $100 \mu \mathrm{M}$ levamisole. Images were captured using a Leica HCX PL APO $40 \times / 0.85$ CORR CS objective or an HC PL APO $63 \times 10.40$ CS objective on a Leica TCS-SP5 confocal microscope. The length of ASER sensory cilium was measured by simple neurite tracer plugin of ImageJ. All depicted representative fluorescence images were Z-stacked.

\section{Electrophysiology}

We synthesized capped CLH-1 cRNAs using T7 mMESSAGE mMACHINE kit (Ambion) and purified by lithium chloride precipitation. cRNA was quantified by spectroscopy. Oocytes from $X$. laevis were prepared as follows. We anesthetized female $X$. laevis in cold MS-222 solution, and excised lobes of ovaries from a small incision made in the posterior ventral side. Oocytes were obtained by defolliculation; incubation of ovaries in $0.2 \%$ collagenase (Wako) in modified Barth's solution (MBSH; $88 \mathrm{mM}$ $\mathrm{NaCl}, 1 \mathrm{mM} \mathrm{KCl}, 2.4 \mathrm{mM} \mathrm{NaHCO} 3,0.82 \mathrm{mM} \mathrm{MgSO}_{4}, 0.33 \mathrm{mM} \mathrm{Ca}\left(\mathrm{NO}_{3}\right)_{2}, 0.41 \mathrm{mM} \mathrm{CaCl}, 10 \mathrm{mM}$ $\mathrm{N}$-(2-hydroxyethyl)piperazine- $N^{\prime}-2$-ethanesulfonic acid (HEPES, pH 7.6)) for 2 to $5 \mathrm{hr}$. Oocytes were washed and incubated at $16^{\circ} \mathrm{C}$ in $\mathrm{MBSH}$ with $100 \mathrm{U} / \mathrm{mL}$ penicillin and $0.1 \mathrm{mg} / \mathrm{mL}$ streptomycin overnight. Thereafter, oocytes were injected with cRNA for a final amount of $50 \mathrm{ng} /$ oocyte. Oocytes were incubated in $\mathrm{MBSH}$ at $16^{\circ} \mathrm{C}$ for 2 days before recording. Currents were measured using a twoelectrode voltage-clamp amplifier Oocyte Clamp OC-725C (Warner) at ambient temperature $\left(22^{\circ} \mathrm{C}\right)$. Electrodes (0.3-1 $\mathrm{M} \Omega$ ) were filled with $3 \mathrm{M} \mathrm{KCl}$, and then oocytes were perfused in an extracellular solution buffer with the following composition (in $\mathrm{mM}$ ): $100 \mathrm{NaCl}, 2 \mathrm{KCl}, 1 \mathrm{CaCl}_{2}, 2 \mathrm{MgCl}_{2}, 10$

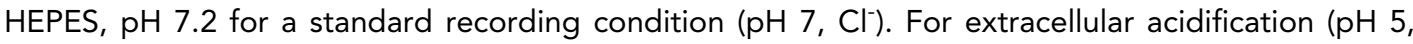
$\left.\mathrm{Cl}^{-}\right), 10 \mathrm{mM}$ of 2-( $\mathrm{N}$-morpholino)ethanesulfonic acid ( $\left.\mathrm{pH} 5.5\right)$ was used instead of $10 \mathrm{mM}$ HEPES. To observe permeability of $\mathrm{HCO}_{3}^{-}\left(\mathrm{pH} 7, \mathrm{HCO}_{3}^{-}\right), 85 \mathrm{mM} \mathrm{NaCl}$ was replaced with an equimolar amount 
of $\mathrm{NaHCO}_{3}$ (Grant et al., 2015). For data acquisition and analysis, pCLAMP suite of programs (Molecular Devices) were used.

\section{In vivo calcium imaging and chloride imaging}

Ratiometric fluorescence reporters Yellow Cameleon 2.6 and SuperClomeleon were used for calcium and chloride imaging, respectively. We found no obvious defects in salt chemotaxis of the animals that carried these reporters in wild-type background. Imaging experiments were performed as described (Iwata et al., 2011; Kunitomo et al., 2013) with minor modifications. Worms were cultivated on standard NGM seeded with OP50 until adulthood and further incubated for $6 \mathrm{hr}$ at distinct salt concentrations with or without food. Worms were then trapped in a polydimethylsiloxane (PDMS) microfluidics device (Chronis et al., 2007), and $\mathrm{NaCl}$ concentration steps were delivered to the animals' nose tip by switching imaging solutions ( $25 \mathrm{mM}$ potassium phosphate ( $\mathrm{pH} 6.0), 1 \mathrm{mM}$ $\mathrm{CaCl}_{2}, 1 \mathrm{mM} \mathrm{MgSO}, 0.02 \%$ gelatin, $\mathrm{NaCl}$ at the indicated concentrations and glycerol to adjust their osmolarity to $350 \mathrm{mOsm}$ ). Time-lapse imaging was conducted with a DMI 6000B microscope (Leica) equipped with an HCX PL APO 63x objective (NA, 1.40), L5 filter set (a combination of 430/ $40 \mathrm{~nm}$ band-path excitation filter and a 40\% transmittance ND filter at 535/40 nm dichromatic mirror, Leica), DualView (Filter sets: $505 \mathrm{dcxr}$ with 480/40 nm and 535/40 nm emission filters), and ImagEM EM-CCD camera (Hamamatsu) at two frames per second. All recordings were started $3 \mathrm{~min}$ after mounting to stabilize the light response of animals. Fluorescence intensity of the soma was measured. The region of interest (ROI) was tracked by Track Objects function of Metamorph software (Molecular Devices). For each frame, fluorescence intensity of the ROI was calculated by subtracting the background intensity (averaged fluorescence intensity adjacent to the ROI) from the average intensity of the ROI. The ratio of YFP/CFP fluorescence was referred to as $R$. $R_{0}$ was defined as the average of $R$ over 50 frames ( $25 \mathrm{~s}$ ) prior to stimulation, and the fluorescence intensity ratio relative to $R_{0}\left(R / R_{0}\right)$ were calculated for a series of images. For traces, $R / R_{0}$ was averaged for all worms at each time point. The average $R / R_{0}$ change of SuperClomeleon was calculated as the difference between the value of last $10 \mathrm{~s}$ during $25 \mathrm{mM}$ salt stimulus and that just prior to salt concentration change (Figure 4f; [averaged $R / R_{0}$ during $\mathrm{B}$ ] - [averaged $R / R_{0}$ during $\mathrm{A}$ ]). The average $R / R_{0}$ change of YC2.6 (e.g. Figure $5 \mathrm{~b}$ ) was calculated as the difference between the highest value of $10 \mathrm{~s}$ moving average of $R / R 0$ during $25 \mathrm{mM} \mathrm{NaCl}$ stimulus $\left(R / R_{0}\right.$ peak) and $10 \mathrm{~s}$ average of $R / R_{0}$ just prior to corresponding $\mathrm{NaCl}$ decrease (e.g. [averaged $R / R_{0}$ peak of $\mathrm{B}$ ] - [averaged $R / R_{0}$ during A] for the 1 st, [averaged $R / R_{0}$ peak of E] - [averaged $R / R_{0}$ during D] for the 2 nd, [averaged $R / R_{0}$ peak of $H$ ] - [averaged $R / R_{0}$ during $G$ ] for the $3 r d$ in Figure $5 a$ ). Note that the time point used for $R / R_{0}$ peak differs between individuals because rise speed was different between clh-1 genotypes. $R / R_{0}$ decay1 (e.g. Figure 5-figure supplement 1a) was calculated as the difference between the $R / R_{0}$ peak and the average of last $10 \mathrm{sec} R / R_{0}$ during $25 \mathrm{mM} \mathrm{NaCl}$ stimulus (therefore just prior to $50 \mathrm{mM} \mathrm{NaCl}$ upstep; e.g. [averaged $R / R_{0}$ during $\mathrm{B}$ ] - [averaged $R / R_{0}$ peak of C] for the 1 st, [averaged $R / R_{0}$ during $E$ ] - [averaged $R / R_{0}$ peak of F] for the 2 nd, [averaged $R / R_{0}$ during $\mathrm{H}$ ] - [averaged $R / R_{0}$ peak of I] for the $3 \mathrm{rd}$, in Figure 5a). $R / R_{0}$ decay2 (e.g. Figure 5-figure supplement $1 \mathrm{~b}$ ) was calculated as the difference between the average of last $10 \mathrm{sec} R / R_{0}$ during $25 \mathrm{mM} \mathrm{NaCl}$ stimulus and the average of last $10 \mathrm{sec} R / R_{0}$ during the following $50 \mathrm{mM} \mathrm{NaCl}$ period (e.g. [averaged $R / R_{0}$ during C] - [averaged $R / R_{0}$ during $D$ ] for the 1 st, [averaged $R / R_{0}$ during F] - [averaged $R / R_{0}$ during G] for the 2nd, in Figure 5a).

\section{Quantification of the expression level of the clh-1 promoter}

Worms that carry clh-1p::nls4::mTFP and gcy-5p::nls4::mCherry were cultivated on standard NGM seeded with OP50 until adulthood. One-day adults were further incubated at $0 \mathrm{mM}$ or $100 \mathrm{mM} \mathrm{NaCl}$ with food for $6 \mathrm{hr}$ if needed. Naive (directly prepared from the cultivation plate) or different salt concentration-experienced animals were mounted on 5\% agar and anesthetized by $10 \mathrm{mM} \mathrm{NaN}_{3}$. Images were captured using a Leica HC PL APO 10×/0.40 CS objective on a Leica TCS-SP5 confocal microscope. The expression level of clh-1 in ASER of each animal was determined as described previously with modifications (Nagashima et al., 2019). The fluorescence intensity ratio of mTFP to $\mathrm{mCherry}$ (mTFP/mCherry) from the confocal image that gave largest area of ASER nucleus was defined as clh-1 expression level. 


\section{Quantitative analysis of animals' behavior}

Quantitative behavior analysis was conducted as described previously with modifications (Jang et al., 2019; Kunitomo et al., 2013). Briefly, animals and chemotaxis assay plates were prepared as described above in salt chemotaxis assay except that $\mathrm{NaN}_{3}$ was omitted. To reduce the chance of collision of worms, only 30-50 worms were placed. Images of whole assay plate were acquired for $15 \mathrm{~min}$ at one frame per second. Probability of pirouette and curving rate were calculated as previously described (Jang et al., 2019). Pirouette index was defined as the difference of pirouette probability between negative $\mathrm{dC} / \mathrm{d}$ t rank and positive $\mathrm{d} C / \mathrm{d} t$ rank. Weathervane index was defined as the slope of the regression line in relationship between $\mathrm{NaCl}$ gradient in normal direction and the curving rate. We exploited data in the range of -0.3 to 0.3 for $\mathrm{dC} / \mathrm{d}$ t and -2 to 2 for $\mathrm{dC} / \mathrm{d} n$ because both pirouette probability and curving rate converged toward zero with high variability in the range of highly negative or positive $d C / d t$ or $d C / d n$ range, probably due to small number of data points at the gradient peaks. We calculated data of each plate, then showed average and s. e.m. in figures. Data from 40 to $540 \mathrm{~s}$ were used to calculate the behavioral parameters because trajectories of worms were highly interrupted by collision of worms before $40 \mathrm{~s}$.

\section{Calcium imaging of AIB in freely moving animal}

Animals expressed GCaMP6s and mCherry in AIB (JN3329, see Supplementary file 1). Worms were cultivated on standard NGM until young adulthood and further incubated overnight on NGM plates with $0 \mathrm{mM} \mathrm{NaCl}$. A total of $20 \sim 25$ worms were washed out from the plate, and injected into a PDMS microfluidic device (Albrecht and Bargmann, 2011). After few minutes of acclimation to the PDMS environment under continuous flow of imaging solution without $\mathrm{NaCl}$ (see In vivo calcium imaging and chloride imaging for composition), a $25 \mathrm{mM} \mathrm{NaCl}$ up-step was delivered to worms. Bright-field images for locomotion analysis were acquired at 33 frames per second and fluorescence images for AIB activity were acquired at 4 frames per second with a BX51 microscope (Olympus) equipped with a halogen light source (U-LH100IR), a motorized stage (HV-STU02- 1, HawkVision), an LMPlanF1 5x objective (NA, 0.13), U-25ND25 (Olympus), a combination of 480/40 nm band-path excitation filter and a $25 \%$ transmittance ND filter at 505/40 nm dichromatic mirror (Leica), DualView (Filter sets: $565 \mathrm{dcxr}$ with 520/30 nm and 630/50 nm emission filters), and a CCD camera (GRASc3K2M-C, Point Grey Research). Tracking of particular animal was performed using a Linux-based software (Satoh et al., 2014). After subtracting background, fluorescence intensity of GCaMP6s was normalized by that of mCherry. Average fluorescence intensity over 50 frames (12.5 s) prior to stimulation was set as $F_{0}$ and the fluorescence intensity relative to $F_{0}\left(F / F_{0}\right)$ were calculated for a series of images. For traces, $F / F_{0}$ was averaged for all worms at each time point. The $F / F_{O}$ change in response to stimulation was calculated as the difference of averaged $F / F_{0}$ between 125 to 175 frames for peak and 51 to 100 frames for baseline (i.e. [averaged $F / F_{0}$ during $B$ ] - [averaged $F / F_{0}$ during $A$ ] in Figure 6e). Reversal was defined as backward movement whose velocity was less than $0 \mu \mathrm{m} / \mathrm{s}$ (Figure 6-figure supplement 2, colored in red). In Figure 6d, animals those showed obvious reversal response (reversed longer than 35 frames (1 s) within 501 frames (15 s) after salt up-step stimulus at velocity less than $-100 \mu \mathrm{m} / \mathrm{s}$ ) were counted as 'reversed animals' to exclude short spontaneous reversals that occur independent of salt stimulus (Figure 6-figure supplement 2, short red bouts).

\section{Data analyses}

The sample sizes were experimentally determined, with referring to those previously reported. Repeats of most experiments were performed in 3-6 separate days. Statistical analyses were performed using Prism v.5 (GraphPad software, San Diego, CA).

\section{Acknowledgements}

We thank the Caenorhabditis Genetics Center (CGC) and the National BioResource Project (NBRP) for strains; Ishihara T for pDEST-nls-YC2.60, Oka Y for electrophysiology setup and technical assistance, Chronis N, Albrecht D and Bargmann C for design of microfluidics chips. We also thank members of the lino laboratory members for helpful comments and discussion. 


\section{Additional information}

Competing interests

Yuichi lino: Reviewing editor, eLife. The other authors declare that no competing interests exist.

Funding

\begin{tabular}{|c|c|c|}
\hline Funder & Grant reference number & Author \\
\hline $\begin{array}{l}\text { Japan Society for the Promo- } \\
\text { tion of Science }\end{array}$ & $\begin{array}{l}\text { Grant-in-Aid for Scientific } \\
\text { Research (S) JP17H06113 }\end{array}$ & Yuichi lino \\
\hline $\begin{array}{l}\text { Japan Science and Technology } \\
\text { Agency }\end{array}$ & CREST JP17H06113 & Yuichi lino \\
\hline $\begin{array}{l}\text { Japan Society for the Promo- } \\
\text { tion of Science }\end{array}$ & $\begin{array}{l}\text { Grants-in-Aid for Innovative } \\
\text { Area "Artificial Intelligence } \\
\text { and Brain Science" } \\
19 \mathrm{H} 04980\end{array}$ & Yuichi lino \\
\hline University of Tokyo & $\begin{array}{l}\text { Center for Integrative } \\
\text { Science of Human Behavior } \\
\text { (CiSHuB) }\end{array}$ & Yuichi lino \\
\hline $\begin{array}{l}\text { Japan Society for the Promo- } \\
\text { tion of Science }\end{array}$ & $\begin{array}{l}\text { Grant-in-Aid for Scientific } \\
\text { Research }\end{array}$ & Hirofumi Sato \\
\hline $\begin{array}{l}\text { Japan Society for the Promo- } \\
\text { tion of Science }\end{array}$ & $\begin{array}{l}\text { Grants-in-Aid for Innovative } \\
\text { Area } 18 \mathrm{H} 04881\end{array}$ & Shinji Kanda \\
\hline $\begin{array}{l}\text { Japan Society for the Promo- } \\
\text { tion of Science }\end{array}$ & $\begin{array}{l}\text { Grant-in-Aid for challenging } \\
\text { Exploratory Research } \\
18 \text { K19323 }\end{array}$ & Shinji Kanda \\
\hline $\begin{array}{l}\text { Japan Society for the Promo- } \\
\text { tion of Science }\end{array}$ & $\begin{array}{l}\text { Grant-in-Aid for Scientific } \\
\text { Research } 19 K 06952\end{array}$ & Hirofumi Kunitomo \\
\hline $\begin{array}{l}\text { Salt Science Research Founda- } \\
\text { tion }\end{array}$ & No. 1728 and No. 2043 & Hirofumi Kunitomo \\
\hline $\begin{array}{l}\text { Japan Society for the Promo- } \\
\text { tion of Science }\end{array}$ & $\begin{array}{l}\text { Grant-in-Aid for Young } \\
\text { Scientists 19K1628 }\end{array}$ & Hirofumi Sato \\
\hline
\end{tabular}

The funders had no role in study design, data collection and interpretation, or the decision to submit the work for publication.

Author contributions

Chanhyun Park, Resources, Formal analysis, Validation, Investigation, Visualization, Methodology, Writing - original draft, Writing - review and editing; Yuki Sakurai, Resources, Validation, Investigation; Hirofumi Sato, Resources, Investigation, Methodology; Shinji Kanda, Resources, Supervision, Funding acquisition, Writing - review and editing; Yuichi lino, Conceptualization, Formal analysis, Supervision, Funding acquisition, Writing - review and editing; Hirofumi Kunitomo, Conceptualization, Resources, Supervision, Funding acquisition, Validation, Investigation, Writing - original draft, Writing - review and editing

Author ORCIDs

Chanhyun Park (iD) https://orcid.org/0000-0002-0200-6903

Yuichi lino (Di) http://orcid.org/0000-0002-0936-2660

Hirofumi Kunitomo (iD https://orcid.org/0000-0001-7312-7051

Decision letter and Author response

Decision letter https://doi.org/10.7554/eLife.55701.sa1

Author response https://doi.org/10.7554/eLife.55701.sa2 


\section{Additional files}

Supplementary files

- Supplementary file 1. Strain list.

- Supplementary file 2. Genotyping primer.

- Supplementary file 3. Plasmid list and use.

- Supplementary file 4. Plasmid sequences.

- Supplementary file 5. Statistics.

- Transparent reporting form

Data availability

All data analysed during this study are included in the manuscript and supporting files. Detailed statistical reporting appears in supplementary file 5 . Other source data files have been uploaded to Dryad Digital Repository (https://doi.org/10.5061/dryad.4tmpg4f8c).

The following dataset was generated:

\begin{tabular}{|c|c|c|c|c|}
\hline Author(s) & Year & Dataset title & Dataset URL & $\begin{array}{l}\text { Database and } \\
\text { Identifier }\end{array}$ \\
\hline $\begin{array}{l}\text { Park } C, \text { Sakurai } Y, \\
\text { Sato } H, \text { Kanda } S, \\
\text { lino } Y, \text { Kunitomo } H\end{array}$ & 2020 & $\begin{array}{l}\text { Roles of the } \mathrm{ClC} \text { chloride channel } \\
\mathrm{CLH}-1 \text { in food-associated salt } \\
\text { chemotaxis behavior of } \mathrm{C} \text {. elegans }\end{array}$ & $\begin{array}{l}\text { https://dx.doi.org/10. } \\
\text { 5061/dryad.4tmpg4f8c }\end{array}$ & $\begin{array}{l}\text { Dryad Digital } \\
\text { Repository, } 10.5061 / \\
\text { dryad.4tmpg4f8c }\end{array}$ \\
\hline
\end{tabular}

\section{References}

Accardi A, Walden M, Nguitragool W, Jayaram H, Williams C, Miller C. 2005. Separate ion pathways in a cl-/H+ exchanger. Journal of General Physiology 126:563-570. DOI: https://doi.org/10.1085/jgp.200509417, PMID: 16316975

Accardi A. 2015. Structure and gating of CLC channels and exchangers. The Journal of Physiology 593:41294138. DOI: https://doi.org/10.1113/JP270575, PMID: 26148215

Adachi T, Kunitomo H, Tomioka M, Ohno H, Okochi Y, Mori I, lino Y. 2010. Reversal of salt preference is directed by the insulin/PI3K and gq/PKC signaling in Caenorhabditis elegans. Genetics 186:1309-1319. DOI: https://doi. org/10.1534/genetics.110.119768, PMID: 20837997

Ahnert-Hilger G, Jahn R. 2011. CLC-3 spices up GABAergic synaptic vesicles. Nature Neuroscience 14:405-407. DOI: https://doi.org/10.1038/nn.2786, PMID: 21445064

Albrecht DR, Bargmann Cl. 2011. High-content behavioral analysis of Caenorhabditis elegans in precise spatiotemporal chemical environments. Nature Methods 8:599-605. DOl: https://doi.org/10.1038/nmeth.1630, PMID: 21666667

Arosio D, Ratto GM. 2014. Twenty years of fluorescence imaging of intracellular chloride. Frontiers in Cellular Neuroscience 8:258. DOI: https://doi.org/10.3389/fncel.2014.00258, PMID: 25221475

Bacaj T, Tevlin M, Lu Y, Shaham S. 2008. Glia are essential for sensory organ function in C. elegans. Science 322 : 744-747. DOI: https://doi.org/10.1126/science.1163074, PMID: 18974354

Bargmann $\mathrm{Cl}$, Horvitz HR. 1991. Chemosensory neurons with overlapping functions direct chemotaxis to multiple chemicals in C. elegans. Neuron 7:729-742. DOI: https://doi.org/10.1016/0896-6273(91)90276-6, PMID: 16602 83

Bianchi L. 2006. Culture of embryonic C. elegans cells for electrophysiological and pharmacological analyses. WormBook 1:122.1. DOl: https://doi.org/10.1895/wormbook.1.122.1

Blanz J, Schweizer M, Auberson M, Maier H, Muenscher A, Hübner CA, Jentsch TJ. 2007. Leukoencephalopathy upon disruption of the chloride channel CIC-2. Journal of Neuroscience 27:6581-6589. DOI: https://doi.org/10. 1523/JNEUROSCI.0338-07.2007, PMID: 17567819

BösI MR, Stein V, Hübner C, Zdebik AA, Jordt SE, Mukhopadhyay AK, Davidoff MS, Holstein AF, Jentsch TJ. 2001. Male germ cells and photoreceptors, both dependent on close cell-cell interactions, degenerate upon CIC-2 cl(-) channel disruption. The EMBO Journal 20:1289-1299. DOI: https://doi.org/10.1093/emboj/20.6. 1289, PMID: 11250895

Branicky R, Miyazaki H, Strange K, Schafer WR. 2014. The voltage-gated anion channels encoded by clh-3 regulate egg laying in $C$. elegans by modulating motor neuron excitability. The Journal of Neuroscience 34: 764-775. DOI: https://doi.org/10.1523/JNEUROSCI.3112-13.2014, PMID: 24431435

Braubach OR, Wood HD, Gadbois S, Fine A, Croll RP. 2009. Olfactory conditioning in the zebrafish (Danio rerio). Behavioural Brain Research 198:190-198. DOI: https://doi.org/10.1016/j.bbr.2008.10.044, PMID: 19056431

Brenner S. 1974. The genetics of Caenorhabditis elegans. Genetics 77:71-94. PMID: 4366476 
Chalasani SH, Chronis N, Tsunozaki M, Gray JM, Ramot D, Goodman MB, Bargmann CI. 2007. Dissecting a circuit for olfactory behaviour in Caenorhabditis elegans. Nature 450:63-70. DOI: https://doi.org/10.1038/ nature06292, PMID: 17972877

Charlet-B N, Savkur RS, Singh G, Philips AV, Grice EA, Cooper TA. 2002. Loss of the muscle-specific chloride channel in type 1 myotonic dystrophy due to misregulated alternative splicing. Molecular Cell 10:45-53. DOI: https://doi.org/10.1016/s1097-2765(02)00572-5, PMID: 12150906

Cho CE, Brueggemann C, L'Etoile ND, Bargmann Cl. 2016. Parallel encoding of sensory history and behavioral preference during Caenorhabditis elegans olfactory learning. eLife 5:e14000. DOI: https://doi.org/10.7554/ eLife. 14000

Chronis N, Zimmer M, Bargmann Cl. 2007. Microfluidics for in vivo imaging of neuronal and behavioral activity in Caenorhabditis elegans. Nature Methods 4:727-731. DOI: https://doi.org/10.1038/nmeth1075, PMID: 177047 83

Colbert HA, Bargmann Cl. 1995. Odorant-specific adaptation pathways generate olfactory plasticity in C. elegans. Neuron 14:803-812. DOI: https://doi.org/10.1016/0896-6273(95)90224-4, PMID: 7718242

Dascal N. 2000. Voltage clamp recordings from Xenopus Oocytes. Current Protocols in Neuroscience 10: ns0612s10. DOI: https://doi.org/10.1002/0471142301.ns0612s10

Deidda G, Parrini M, Naskar S, Bozarth IF, Contestabile A, Cancedda L. 2015. Reversing excitatory GABAAR signaling restores synaptic plasticity and memory in a mouse model of down syndrome. Nature Medicine $\mathbf{2 1}$ 318-326. DOI: https://doi.org/10.1038/nm.3827, PMID: 25774849

Denton J, Nehrke K, Yin X, Morrison R, Strange K. 2005. GCK-3, a newly identified Ste20 kinase, binds to and regulates the activity of a cell Cycle-dependent CIC Anion Channel. Journal of General Physiology 125:113125. DOI: https://doi.org/10.1085/jgp.200409215

Duran C, Thompson CH, Xiao Q, Hartzell HC. 2009. Chloride channels: often enigmatic, rarely predictable. Annual Review of Physiology 72:95-121. DOl: https://doi.org/10.1146/annurev-physiol-021909-135811

Dutzler R, Campbell EB, Cadene M, Chait BT, MacKinnon R. 2002. X-ray structure of a ClC chloride channel at 3. 0 A reveals the molecular basis of anion selectivity. Nature 415:287-294. DOI: https://doi.org/10.1038/ 415287a, PMID: 11796999

Dutzler R, Campbell EB, MacKinnon R. 2003. Gating the selectivity filter in CIC chloride channels. Science $\mathbf{3 0 0 :}$ 108-112. DOI: https://doi.org/10.1126/science.1082708, PMID: 12649487

Estévez R, Pusch M, Ferrer-Costa C, Orozco M, Jentsch TJ. 2004. Functional and structural conservation of CBS domains from CLC chloride channels. The Journal of Physiology 557:363-378. DOI: https://doi.org/10.1113/ jphysiol.2003.058453, PMID: 14724190

Fay D, Bender A. 2006. Genetic mapping and manipulation: chapter 4-SNPs: introduction and Two-Point mapping. WormBook: The Online Review of C. elegans Biology 1:1-17. DOI: https://doi.org/10.1895/ wormbook.1.93.1

Feng L, Campbell EB, Hsiung Y, MacKinnon R. 2010. Structure of a eukaryotic CLC transporter defines an intermediate state in the transport cycle. Science 330:635-641. DOI: https://doi.org/10.1126/science.1195230, PMID: 20929736

Fernandes-Rosa FL, Daniil G, Orozco IJ, Göppner C, El Zein R, Jain V, Boulkroun S, Jeunemaitre X, Amar L, Lefebvre H, Schwarzmayr T, Strom TM, Jentsch TJ, Zennaro MC. 2018. A gain-of-function mutation in the CLCN2 chloride channel gene causes primary aldosteronism. Nature Genetics 50:355-361. DOI: https://doi. org/10.1038/s41588-018-0053-8, PMID: 29403012

Goodman MB, Hall DH, Avery L, Lockery SR. 1998. Active currents regulate sensitivity and dynamic range in C. elegans neurons. Neuron 20:763-772. DOI: https://doi.org/10.1016/S0896-6273(00)81014-4, PMID: 9581767

Gordus A, Pokala N, Levy S, Flavell SW, Bargmann Cl. 2015. Feedback from network states generates variability in a probabilistic olfactory circuit. Cell 161:215-227. DOI: https://doi.org/10.1016/j.cell.2015.02.018, PMID: 25772698

Gottfried JA, O'Doherty J, Dolan RJ. 2003. Encoding predictive reward value in human amygdala and orbitofrontal cortex. Science 301:1104-1107. DOI: https://doi.org/10.1126/science.1087919, PMID: 12934011

Gottschalk A, Almedom RB, Schedletzky T, Anderson SD, Yates JR, Schafer WR. 2005. Identification and characterization of novel nicotinic receptor-associated proteins in Caenorhabditis elegans. The EMBO Journal 24:2566-2578. DOI: https://doi.org/10.1038/sj.emboj.7600741, PMID: 15990870

Grant J, Matthewman C, Bianchi L. 2015. A novel mechanism of pH buffering in C. elegans Glia: Bicarbonate Transport via the Voltage-Gated ClC Cl- Channel CLH-1. Journal of Neuroscience 35:16377-16397. DOI: https://doi.org/10.1523/JNEUROSCI.3237-15.2015, PMID: 26674864

Gray JM, Hill JJ, Bargmann Cl. 2005. A circuit for navigation in Caenorhabditis elegans. PNAS 102:3184-3191. DOI: https://doi.org/10.1073/pnas.0409009101

Grimley JS, Li L, Wang W, Wen L, Beese LS, Hellinga HW, Augustine GJ. 2013. Visualization of synaptic inhibition with an optogenetic sensor developed by cell-free protein engineering automation. Journal of Neuroscience 33:16297-16309. DOI: https://doi.org/10.1523/JNEUROSCI.4616-11.2013, PMID: 24107961

Hedgecock EM, Russell RL. 1975. Normal and mutant thermotaxis in the nematode Caenorhabditis elegans. PNAS 72:4061-4065. DOI: https://doi.org/10.1073/pnas.72.10.4061

Hirano Y, Masuda T, Naganos S, Matsuno M, Ueno K, Miyashita T, Horiuchi J, Saitoe M. 2013. Fasting launches CRTC to facilitate long-term memory formation in Drosophila. Science 80:443-446. DOI: https://doi.org/10. 1126/science. 1227170 
Horikawa K, Yamada Y, Matsuda T, Kobayashi K, Hashimoto M, Matsu-ura T, Miyawaki A, Michikawa T, Mikoshiba K, Nagai T. 2010. Spontaneous network activity visualized by ultrasensitive ca(2+) indicators, yellow Cameleon-Nano. Nature Methods 7:729-732. DOI: https://doi.org/10.1038/nmeth.1488, PMID: 20693999

lino Y, Yoshida K. 2009. Parallel use of two behavioral mechanisms for chemotaxis in Caenorhabditis elegans. Journal of Neuroscience 29:5370-5380. DOI: https://doi.org/10.1523/JNEUROSCI.3633-08.2009, PMID: 19403 805

Iwata R, Oda S, Kunitomo H, lino Y. 2011. Roles for class IIA phosphatidylinositol transfer protein in Neurotransmission and behavioral plasticity at the sensory neuron synapses of Caenorhabditis elegans. PNAS 108:7589-7594. DOI: https://doi.org/10.1073/pnas.1016232108, PMID: 21502506

Jang MS, Toyoshima Y, Tomioka M, Kunitomo H, lino Y. 2019. Multiple sensory neurons mediate starvationdependent aversive navigation in Caenorhabditis elegans. PNAS 116:18673-18683. DOI: https://doi.org/10. 1073/pnas.1821716116

Jentsch TJ. 2007. Chloride and the endosomal-lysosomal pathway: emerging roles of CLC chloride transporters. The Journal of Physiology 578:633-640. DOI: https://doi.org/10.1113/jphysiol.2006.124719, PMID: 17110406

Jentsch TJ. 2008. CLC chloride channels and transporters: from genes to protein structure, pathology and physiology. Critical Reviews in Biochemistry and Molecular Biology 43:3-36. DOI: https://doi.org/10.1080/ 10409230701829110, PMID: 18307107

Jeworutzki E, Lagostena L, Elorza-Vidal X, López-Hernández T, Estévez R, Pusch M. 2014. GlialCAM, a CLC-2 cl() channel subunit, activates the slow gate of CLC chloride channels. Biophysical Journal 107:1105-1116. DOI: https://doi.org/10.1016/j.bpj.2014.07.040, PMID: 25185546

Katz M, Corson F, Keil W, Singhal A, Bae A, Lu Y, Liang Y, Shaham S. 2019. Glutamate spillover in C. elegans triggers repetitive behavior through presynaptic activation of MGL-2/mGluR5. Nature Communications 10:113. DOI: https://doi.org/10.1038/s41467-019-09581-4

Kindt KS, Quast KB, Giles AC, De S, Hendrey D, Nicastro I, Rankin CH, Schafer WR. 2007. Dopamine mediates context-dependent modulation of sensory plasticity in C. elegans. Neuron 55:662-676. DOI: https://doi.org/10. 1016/j.neuron.2007.07.023, PMID: 17698017

Kunitomo H, Sato H, Iwata R, Satoh Y, Ohno H, Yamada K, lino Y. 2013. Concentration memory-dependent synaptic plasticity of a taste circuit regulates salt concentration chemotaxis in Caenorhabditis elegans. Nature Communications 4:2210. DOI: https://doi.org/10.1038/ncomms3210, PMID: 23887678

Kunitomo H, lino Y. 2020. Caenorhabditis elegans che-5 is allelic to gcy-22. microPublication Biol 1:000313. DOI: https://doi.org/10.17912/micropub.biology.000313

Kuramochi M, Doi M. 2018. An excitatory/Inhibitory switch from asymmetric sensory neurons defines postsynaptic tuning for a rapid response to $\mathrm{NaCl}$ in Caenorhabditis elegans. Frontiers in Molecular Neuroscience 11:484. DOI: https://doi.org/10.3389/fnmol.2018.00484, PMID: 30687001

Lewis JA, Wu CH, Berg H, Levine JH. 1980. The genetics of levamisole resistance in the nematode Caenorhabditis elegans. Genetics 95:905-928. PMID: 7203008

Li Z, Liu J, Zheng M, Xu XZ. 2014. Encoding of both analog- and digital-like behavioral outputs by one C. elegans interneuron. Cell 159:751-765. DOI: https://doi.org/10.1016/j.cell.2014.09.056, PMID: 25417153

Luo L, Wen Q, Ren J, Hendricks M, Gershow M, Qin Y, Greenwood J, Soucy ER, Klein M, Smith-Parker HK, Calvo AC, Colón-Ramos DA, Samuel AD, Zhang Y. 2014. Dynamic encoding of perception, memory, and movement in a C. elegans chemotaxis circuit. Neuron 82:1115-1128. DOl: https://doi.org/10.1016/j. neuron.2014.05.010, PMID: 24908490

Miyazaki H, Yamada T, Parton A, Morrison R, Kim S, Beth AH, Strange K. 2012. CLC anion channel regulatory phosphorylation and conserved signal transduction domains. Biophysical Journal 103:1706-1718. DOI: https:// doi.org/10.1016/j.bpj.2012.09.001, PMID: 23083714

Nagashima T, lino Y, Tomioka M. 2019. DAF-16/FOXO promotes taste avoidance learning independently of axonal insulin-like signaling. PLOS Genetics 15:e1008297. DOI: https://doi.org/10.1371/journal.pgen.1008297, PMID: 31323047

Nehrke K, Begenisich T, Pilato J, Melvin JE. 2000. Into ion channel and transporter function. Caenorhabditis elegans CIC-type chloride channels: novel variants and functional expression. American Journal of PhysiologyCell Physiology 279:C2052-C2066. DOI: https://doi.org/10.1152/ajpcell.2000.279.6.C2052, PMID: 11078724

O'Doherty JP, Dayan P, Friston K, Critchley H, Dolan RJ. 2003. Temporal difference models and reward-related learning in the human brain. Neuron 38:329-337. DOI: https://doi.org/10.1016/S0896-6273(03)00169-7, PMID: 12718865

Oda S, Tomioka M, lino Y. 2011. Neuronal plasticity regulated by the insulin-like signaling pathway underlies salt chemotaxis learning in Caenorhabditis elegans. Journal of Neurophysiology 106:301-308. DOI: https://doi.org/ 10.1152/jn.01029.2010, PMID: 21525368

Ohno H, Kato S, Naito Y, Kunitomo H, Tomioka M, lino Y. 2014. Role of synaptic phosphatidylinositol 3-kinase in a behavioral learning response in C. elegans. Science 345:313-317. DOI: https://doi.org/10.1126/science. 1250709, PMID: 25035490

Ortiz CO, Faumont S, Takayama J, Ahmed HK, Goldsmith AD, Pocock R, McCormick KE, Kunimoto H, lino Y, Lockery S, Hobert O. 2009. Lateralized gustatory behavior of $C$. elegans is controlled by specific receptor-type guanylyl cyclases. Current Biology 19:996-1004. DOI: https://doi.org/10.1016/j.cub.2009.05.043, PMID: 19523 832

Otis JM, Namboodiri VM, Matan AM, Voets ES, Mohorn EP, Kosyk O, McHenry JA, Robinson JE, Resendez SL, Rossi MA, Stuber GD. 2017. Prefrontal cortex output circuits guide reward seeking through divergent cue encoding. Nature 543:103-107. DOI: https://doi.org/10.1038/nature21376, PMID: 28225752 
Pavlov IP. 1927. Classics in the History of Psychology - Pavlov (1927) Lecture 2: Cond REFLEXES an Investig Physiol Act Cereb CORTEX.

Perkins LA, Hedgecock EM, Thomson JN, Culotti JG. 1986. Mutant sensory cilia in the nematode Caenorhabditis elegans. Developmental Biology 117:456-487. DOI: https://doi.org/10.1016/0012-1606(86)90314-3, PMID: 242 8682

Pfeffer CK, Stein V, Keating DJ, Maier H, Rinke I, Rudhard Y, Hentschke M, Rune GM, Jentsch TJ, Hübner CA. 2009. NKCC1-dependent GABAergic excitation drives synaptic network maturation during early hippocampal development. Journal of Neuroscience 29:3419-3430. DOI: https://doi.org/10.1523/JNEUROSCI.1377-08. 2009, PMID: 19295148

Pierce-Shimomura JT, Faumont S, Gaston MR, Pearson BJ, Lockery SR. 2001. The homeobox gene lim-6 is required for distinct chemosensory representations in C. elegans. Nature 410:694-698. DOI: https://doi.org/10 1038/35070575, PMID: 11287956

Piggott BJ, Liu J, Feng Z, Wescott SA, Xu XZ. 2011. The neural circuits and synaptic mechanisms underlying motor initiation in C. elegans. Cell 147:922-933. DOI: https://doi.org/10.1016/j.cell.2011.08.053, PMID: 2207 8887

Ratté S, Prescott SA. 2011. ClC-2 channels regulate neuronal excitability, not intracellular chloride levels. Journal of Neuroscience 31:15838-15843. DOI: https://doi.org/10.1523/JNEUROSCI.2748-11.2011, PMID: 22049427

Redemann S, Schloissnig S, Ernst S, Pozniakowsky A, Ayloo S, Hyman AA, Bringmann H. 2011. Codon adaptation-based control of protein expression in C. elegans. Nature Methods 8:250-252. DOI: https://doi. org/10.1038/nmeth.1565, PMID: 21278743

Rinke I, Artmann J, Stein V. 2010. ClC-2 voltage-gated channels constitute part of the background conductance and assist chloride extrusion. Journal of Neuroscience 30:4776-4786. DOI: https://doi.org/10.1523/ JNEUROSCI.6299-09.2010, PMID: 20357128

Saeki S, Yamamoto M, lino Y. 2001. Plasticity of chemotaxis revealed by paired presentation of a chemoattractant and starvation in the nematode Caenorhabditis elegans. The Journal of Experimental Biology 204:1757-1764. PMID: 11316496

Sasakura H, Mori I. 2013. Behavioral plasticity, learning, and memory in C. elegans. Current Opinion in Neurobiology 23:92-99. DOI: https://doi.org/10.1016/j.conb.2012.09.005, PMID: 23063296

Satoh Y, Sato H, Kunitomo H, Fei X, Hashimoto K, lino Y. 2014. Regulation of experience-dependent bidirectional chemotaxis by a neural circuit switch in Caenorhabditis elegans. Journal of Neuroscience 34: 15631-15637. DOI: https://doi.org/10.1523/JNEUROSCI.1757-14.2014, PMID: 25411491

Schriever AM, Friedrich T, Pusch M, Jentsch TJ. 1999. CLC chloride channels in Caenorhabditis elegans. The Journal of Biological Chemistry 274:34238-34244. DOI: https://doi.org/10.1074/jbc.274.48.34238, PMID: 10567397

Serrano-Saiz E, Poole RJ, Felton T, Zhang F, De La Cruz ED, Hobert O. 2013. Modular control of glutamatergic neuronal identity in C. elegans by distinct homeodomain proteins. Cell 155:659-673. DOI: https://doi.org/10. 1016/j.cell.2013.09.052, PMID: 24243022

Shindou T, Ochi-Shindou M, Murayama T, Saita E, Momohara Y, Wickens JR, Maruyama IN. 2019. Active propagation of dendritic electrical signals in C. elegans. Scientific Reports 9:e40158-9. DOI: https://doi.org/10. 1038/s41598-019-40158-9

Singhvi A, Liu B, Friedman CJ, Fong J, Lu Y, Huang X-Y, Shaham S. 2016. A Glial K/Cl Transporter Controls Neuronal Receptive Ending Shape by Chloride Inhibition of an rGC. Cell 165:936-948. DOI: https://doi.org/10. 1016/j.cell.2016.03.026

Smith HK, Luo L, O'Halloran D, Guo D, Huang XY, Samuel AD, Hobert O. 2013. Defining specificity determinants of cGMP mediated gustatory sensory transduction in Caenorhabditis elegans. Genetics 194:885-901.

DOI: https://doi.org/10.1534/genetics.113.152660, PMID: 23695300

Spencer WC, Zeller G, Watson JD, Henz SR, Watkins KL, McWhirter RD, Petersen S, Sreedharan VT, Widmer C, Jo J, Reinke V, Petrella L, Strome S, Von Stetina SE, Katz M, Shaham S, Ratsch G, Miller DM. 2011. A spatial and temporal map of C. elegans gene expression. Genome Research 21:325-341. DOI: https://doi.org/10.1101/gr. 114595.110

Staley KJ, Soldo BL, Proctor WR. 1995. Ionic mechanisms of neuronal excitation by inhibitory GABAA receptors. Science 269:977-981. DOI: https://doi.org/10.1126/science.7638623, PMID: 7638623

Staley K, Smith R, Schaack J, Wilcox C, Jentsch TJ. 1996. Alteration of GABAA receptor function following gene transfer of the CLC-2 chloride channel. Neuron 17:543-551. DOI: https://doi.org/10.1016/S0896-6273(00) 80186-5, PMID: 8816717

Stauber T, Weinert S, Jentsch TJ. 2012. Cell biology and physiology of CLC chloride channels and transporters. Comprehensive Physiology 2:1701-1744. DOI: https://doi.org/10.1002/cphy.c110038, PMID: 23723021

Stölting G, Teodorescu G, Begemann B, Schubert J, Nabbout R, Toliat MR, Sander T, Nürnberg P, Lerche H, Fahlke C. 2013. Regulation of CIC-2 gating by intracellular ATP. Pflügers Archiv - European Journal of Physiology 465:1423-1437. DOI: https://doi.org/10.1007/s00424-013-1286-0

Stölting G, Fischer M, Fahlke C. 2014. ClC-1 and ClC-2 form hetero-dimeric channels with novel protopore functions. Pflügers Archiv - European Journal of Physiology 466:2191-2204. DOI: https://doi.org/10.1007/ s00424-014-1490-6

Summers PJ, Layne RM, Ortega AC, Harris GP, Bamber BA, Komuniecki RW. 2015. Multiple sensory inputs are extensively integrated to modulate nociception in C. elegans. Journal of Neuroscience 35:10331-10342. DOI: https://doi.org/10.1523/JNEUROSCI.0225-15.2015, PMID: 26180208 
Suzuki H, Thiele TR, Faumont S, Ezcurra M, Lockery SR, Schafer WR. 2008. Functional asymmetry in Caenorhabditis elegans taste neurons and its computational role in chemotaxis. Nature 454:114-117. DOI: https://doi.org/10.1038/nature06927, PMID: 18596810

Thiemann A, Gründer S, Pusch M, Jentsch TJ. 1992. A chloride channel widely expressed in epithelial and nonepithelial cells. Nature 356:57-60. DOI: https://doi.org/10.1038/356057a0

Ventimiglia D, Bargmann Cl. 2017. Diverse modes of synaptic signaling, regulation, and plasticity distinguish two classes of C. elegans glutamatergic neurons. eLife 6:e31234. DOl: https://doi.org/10.7554/eLife.31234

Wallace SW, Singhvi A, Liang Y, Lu Y, Shaham S. 2016. PROS-1/Prospero is a major regulator of the Glia-Specific secretome controlling Sensory-Neuron shape and function in C. elegans. Cell Reports 15:550-562. DOI: https://doi.org/10.1016/j.celrep.2016.03.051, PMID: 27068465

Wang L, Sato H, Satoh Y, Tomioka M, Kunitomo H, lino Y. 2017. A gustatory neural circuit of Caenorhabditis elegans Generates Memory-Dependent Behaviors in Na ${ }^{+}$Chemotaxis. The Journal of Neuroscience 37:20972111. DOI: https://doi.org/10.1523/JNEUROSCI.1774-16.2017, PMID: 28126744

Wang K, Preisler SS, Zhang L, Cui Y, Missel JW, Grønberg C, Gotfryd K, Lindahl E, Andersson M, Calloe K, Egea PF, Klaerke DA, Pusch M, Pedersen PA, Zhou ZH, Gourdon P. 2019. Structure of the human CIC-1 chloride channel. PLOS Biology 17:e3000218. DOI: https://doi.org/10.1371/journal.pbio.3000218, PMID: 31022181

Ward S, Thomson N, White JG, Brenner S. 1975. Electron microscopical reconstruction of the anterior sensory anatomy of the nematode Caenorhabditis elegans.?. The Journal of Comparative Neurology 160:313-337. DOI: https://doi.org/10.1002/cne.901600305, PMID: 1112927

Ware RW, Clark D, Crossland K, Russell RL. 1975. The nerve ring of the nematode Caenorhabditis elegans: sensory input and motor output. The Journal of Comparative Neurology 162:71-110. DOI: https://doi.org/10. 1002/cne.901620106

Watteyne J, Peymen K, Van der Auwera P, Borghgraef C, Vandewyer E, Van Damme S, Rutten I, Lammertyn J, Jelier R, Schoofs L, Beets I. 2020. Neuromedin U signaling regulates retrieval of learned salt avoidance in a $C$. elegans gustatory circuit. Nature Communications 11:1-16. DOI: https://doi.org/10.1038/s41467-020-15964-9, PMID: 32350283

Wicks SR, Yeh RT, Gish WR, Waterston RH, Plasterk RH. 2001. Rapid gene mapping in Caenorhabditis elegans using a high density polymorphism map. Nature Genetics 28:160-164. DOI: https://doi.org/10.1038/88878, PMID: 11381264

Winter Y, Stich KP. 2005. Foraging in a complex naturalistic environment: capacity of spatial working memory in flower bats. Journal of Experimental Biology 208:539-548. DOI: https://doi.org/10.1242/jeb.01416, PMID: 15671342

Yamamoto T, Shimojima K, Sangu N, Komoike Y, Ishii A, Abe S, Yamashita S, Imai K, Kubota T, Fukasawa T, Okanishi T, Enoki H, Tanabe T, Saito A, Furukawa T, Shimizu T, Milligan CJ, Petrou S, Heron SE, Dibbens LM, et al. 2015. Single nucleotide variations in CLCN6 identified in patients with benign partial epilepsies in infancy and/or febrile seizures. PLOS ONE 10:e0118946. DOI: https://doi.org/10.1371/journal.pone.0118946, PMID: 25794116

Yoshida A, Nakano S, Suzuki T, Ihara K, Higashiyama T, Mori I. 2016. A glial K(+) /Cl(-) cotransporter modifies temperature-evoked dynamics in Caenorhabditis elegans sensory neurons. Genes, Brain, and Behavior 15:429440. DOI: https://doi.org/10.1111/gbb.12260, PMID: 26463820

Zou W, Fu J, Zhang H, Du K, Huang W, Yu J, Li S, Fan Y, Baylis HA, Gao S, Xiao R, Ji W, Kang L, Xu T. 2018. Decoding the intensity of sensory input by two glutamate receptors in one $C$. elegans interneuron. Nature Communications 9:e06819-5. DOI: https://doi.org/10.1038/s41467-018-06819-5 